\title{
197 CANDIDATES AND 104 VALIDATED PLANETS IN K2's FIRST FIVE FIELDS
}

\author{
Ian J. M. Crossfield ${ }^{1,28,30}$, David R. Ciardi ${ }^{2}$, Erik A. Petigura ${ }^{3,31}$, Evan Sinukoff ${ }^{4,32}$, Joshua E. Schlieder ${ }^{2,5,33}$, \\ Andrew W. Howard ${ }^{4}$, Charles A. Beichman ${ }^{2}$, Howard Isaacson ${ }^{6}$, Courtney D. Dressing ${ }^{3,30}$, Jessie L. Christiansen ${ }^{2}$, \\ Benjamin J. Fulton ${ }^{4,34}$, Sébastien Lépine ${ }^{7}$, Lauren Weiss ${ }^{6}$, Lea Hirsch ${ }^{6}$, John Livingston ${ }^{8}$, Christoph Baranec ${ }^{9}$, \\ Nicholas M. LaW ${ }^{10}$, Reed Riddle ${ }^{11}$, Carl Ziegler ${ }^{10}$, Steve B. Howell ${ }^{5}$, Elliott Horch ${ }^{12}$, Mark Everett ${ }^{13}$, \\ Johanna Teske $^{14}$, Arturo O. Martinez ${ }^{7,15}$, Christian Obermeier ${ }^{16}$, BJörn Benneke ${ }^{3}$, Nic Scott ${ }^{17}$, Niall Deacon ${ }^{18}$, \\ Kimberly M. Aller ${ }^{4}$, Brad M. S. Hansen ${ }^{19}$, Luigi Mancini ${ }^{16}$, Simona Ciceri ${ }^{16,20}$, Rafael Brahim ${ }^{21,22}$, Andrés Jordán ${ }^{21,22}$, \\ Heather A. Knutson ${ }^{3}$, Thomas Henning ${ }^{16}$, Michaël Bonnefoy ${ }^{23,24}$, Michael C. Liu ${ }^{4}$, Justin R. Crepp ${ }^{25}$, \\ Joshua Lothringer ${ }^{1}$, Phil Hinz ${ }^{26}$, VAnessa Bailey ${ }^{26,27}$, Andrew Skemer ${ }^{26,28}$, and Denis Defrere ${ }^{23,24,29}$ \\ ${ }^{1}$ Lunar \& Planetary Laboratory, University of Arizona, 1629 E. University Boulevard., Tucson, AZ, USA \\ ${ }_{3}^{2}$ NASA Exoplanet Science Institute, California Institute of Technology, Pasadena, CA, USA \\ ${ }^{3}$ Geological and Planetary Sciences, California Institute of Technology, Pasadena, CA, USA \\ ${ }^{4}$ Institute for Astronomy, University of Hawai'i at Mānoa, Honolulu, HI, USA \\ ${ }^{5}$ NASA Ames Research Center, Moffett Field, CA, USA \\ ${ }^{6}$ Astronomy Department, University of California, Berkeley, CA, USA \\ ${ }^{7}$ Department of Physics and Astronomy, Georgia State University, GA, USA \\ ${ }^{8}$ Department of Astronomy, The University of Tokyo, 7-3-1 Bunkyo-ku, Tokyo 113-0033, Japan \\ ${ }^{10}$ Department of Physics and Astronomy, University of North Carolina at Chapel Hill, Chapel Hill, NC, USA \\ ${ }^{11}$ Division of Physics, Mathematics, and Astronomy, California Institute of Technology, Pasadena, CA, USA \\ ${ }^{12}$ Department of Physics, Southern Connecticut State University, New Haven, CT, USA \\ ${ }^{13}$ National Optical Astronomy Observatory, Tucson, AZ, USA \\ ${ }^{14}$ Carnegie Department of Terrestrial Magnetism, Washington, DC, USA \\ ${ }^{15}$ Department of Astronomy, San Diego State University, San Diego, CA, USA \\ 16 Max Planck Institut für Astronomie, Heidelberg, Germany
${ }^{17}$ Sydney Institute of Astronomy, The University of Sydney, Redfern, Australia \\ ${ }^{18}$ Centre for Astrophysics Research, University of Hertfordshire, UK \\ ${ }^{19}$ Department of Physics \& Astronomy and Institute of Geophysics \& Planetary Physics, University of California Los Angeles, Los Angeles, CA, USA \\ ${ }^{20}$ Department of Astronomy, Stockholm University, SE-106 91 Stockholm, Sweden \\ ${ }^{21}$ Millennium Institute of Astrophysics, Av. Vicuña Mackenna 4860, 7820436 Macul, Santiago, Chile \\ ${ }^{22}$ Instituto de Astrofísica, Facultad de Física, Pontificia Universidad Católica de Chile, Av. Vicuña Mackenna 4860, 7820436 Macul, Santiago, Chile \\ ${ }^{23}$ Univ. Grenoble Alpes, IPAG, F-38000, Grenoble, France \\ ${ }^{24}$ CNRS, IPAG, F-38000, Grenoble, France \\ 25 Department of Physics, University of Notre Dame, 225 Nieuwland Science Hall, Notre Dame, IN, USA \\ ${ }^{26}$ Steward Observatory, The University of Arizona, Tucson, AZ, USA \\ ${ }^{27}$ Kavli Institute for Particle Astrophysics and Cosmology, Stanford University, Stanford, CA, USA \\ ${ }^{28}$ Department of Astronomy, University of California, Santa Cruz, Santa Cruz, CA, USA \\ ${ }^{29}$ Departement d'Astrophysique, Geophysique et Oceanographie, Universite de Liege, B-4000 Sart Tilman, Belgium \\ Received 2016 April 1; revised 2016 June 20; accepted 2016 June 20; published 2016 September 2
}

\begin{abstract}
We present 197 planet candidates discovered using data from the first year of the NASA K2 mission (Campaigns $0-4$ ), along with the results of an intensive program of photometric analyses, stellar spectroscopy, high-resolution imaging, and statistical validation. We distill these candidates into sets of 104 validated planets (57 in multi-planet systems), 30 false positives, and 63 remaining candidates. Our validated systems span a range of properties, with median values of $R_{P}=2.3 R_{\oplus}, P=8.6$ days, $T_{\text {eff }}=5300 \mathrm{~K}$, and $K p=12.7 \mathrm{mag}$. Stellar spectroscopy provides precise stellar and planetary parameters for most of these systems. We show that $K 2$ has increased by $30 \%$ the number of small planets known to orbit moderately bright stars $\left(1-4 R_{\oplus}, K p=9-13 \mathrm{mag}\right)$. Of particular interest are 76 planets smaller than $2 R_{\oplus}, 15$ orbiting stars brighter than $K p=11.5$ mag, 5 receiving Earth-like irradiation levels, and several multi-planet systems-including 4 planets orbiting the M dwarf K2-72 near mean-motion resonances. By quantifying the likelihood that each candidate is a planet we demonstrate that our candidate sample has an overall false positive rate of $15 \%-30 \%$, with rates substantially lower for small candidates $\left(<2 R_{\oplus}\right)$ and larger for candidates with radii $>8 R_{\oplus}$ and/or with $P<3$ days. Extrapolation of the current planetary yield suggests that $K 2$ will discover between 500 and 1000 planets in its planned four-year mission, assuming sufficient follow-up resources are available. Efficient observing and analysis, together with an organized and coherent follow-up strategy, are essential for maximizing the efficacy of planet-validation efforts for K2, TESS, and future large-scale surveys.
\end{abstract}

\footnotetext{
${ }^{30}$ NASA Sagan Fellow.

31 Hubble Fellow.

32 NSERC Postgraduate Research Fellow.

33 NASA Postdoctoral Program Fellow.

${ }^{34}$ NSF Graduate Research Fellow.
} 
Key words: catalogs - planets and satellites: fundamental parameters - planets and satellites: general - techniques: high angular resolution - techniques: photometric - techniques: spectroscopic

Supporting material: machine-readable tables

\section{INTRODUCTION}

Planets that transit their host stars offer unique opportunities to characterize planetary masses, radii, and densities; atmospheric composition, circulation, and chemistry; dynamical interactions in multi-planet systems; and orbital alignments and evolution, to name just a few aspects of interest. Transiting planets are also the most common type of exoplanet known, thanks in large part to NASA's Kepler spacecraft. Data from Kepler's initial four-year survey revealed over 4000 candidate exoplanets and many confirmed and validated planets ${ }^{35}$ (e.g., Coughlin et al. 2016; Morton et al. 2016). A majority of all exoplanets known today were discovered by Kepler. After the spacecraft's loss of a second reaction wheel in 2014, the mission was renamed $K 2$ and embarked on a new survey of the ecliptic plane, divided into campaigns of roughly 80 days each (Howell et al. 2014). In terms of survey area, temporal coverage, and data release strategy, $K 2$ provides a natural transition from Kepler to the TESS mission (Ricker et al. 2014). Kepler observed 1/400th of the sky for four years (initially with a default proprietary period), while TESS will observe nearly the entire sky for $\geqslant 27$ days,${ }^{36}$ with no default proprietary period.

In its brief history $K 2$ has already made many new discoveries. The mission's data have helped to reveal oscillations in variable stars (Angus et al. 2016) and discovered eclipsing binaries (LaCourse et al. 2015; Armstrong et al. 2016; David et al. 2016a), supernovae (Zenteno et al. 2015), large numbers of planet candidates (Foreman-Mackey et al. 2015; Adams et al. 2016; Vanderburg et al. 2016), and a growing sample of validated and/or confirmed planets (e.g., Vanderburg \& Johnson 2014; Crossfield et al. 2015; Huang et al. 2015; Montet et al. 2015; Sanchis-Ojeda et al. 2015; Sinukoff et al. 2016). Here, we report our identification and follow-up observations of 197 candidate planets using K2 data. Using all available observations and a robust statistical framework, we validate 104 of these as true, bona fide planets, and for the remaining systems we discriminate between obvious false positives and a remaining subset of plausible candidates suitable for further follow-up.

In Section 2 we review our target sample, photometry and transit search, and initial target vetting. Section 3 describes our supporting ground-based observations (stellar spectroscopy and high-resolution imaging; HRI), while Section 4 describes our derivation of stellar parameters. These are followed by our intensive transit light curve analysis in Section 5, the assessment of FPPs for our candidates in Section 6, and a discussion of the results, interesting trends, and noteworthy individual systems in Section 7. Finally, we conclude and summarize in Section 8.

\footnotetext{
35 We distinguish "confirmed" systems (with measured masses) from "validated" systems (whose planetary nature has been statistically demonstrated, e.g., with false positive probability (FPP) $<1 \%$ ).

36 Smaller fractions of the sky will be observed for up to 351 days.
}

\section{K2 TARGETS AND PHOTOMETRY}

\subsection{Target Selection}

In the analysis that follows we use data from all $K 2$ targets (not just those in our own General Observer proposals ${ }^{37}$ ). Huber et al. (2016) present the full distribution of stellar types observed by $K 2$. For completeness we describe here our target selection strategy, which has successfully proposed for thousands of FGK and M dwarfs through two parallel efforts.

We select our FGK stellar sample from the all-sky TESS Dwarf Catalog (TDC; Stassun et al. 2014). The TDC consists of 3 million F5-M5 candidate stars selected from 2MASS and cross-matched with the NOMAD, Tycho-2, Hipparcos, APASS, and UCAC4 catalogs to obtain photometric colors, proper motions, and parallaxes. We remove giant stars based on reduced proper motion versus $J-H$ color (see Collier Cameron et al. 2007), and generate a magnitude-limited dwarf star sample from the merged TDC/EPIC by requiring $K p<14$ mag for these FGK stars. We impose an anti-crowding criterion and remove all targets with a second star in EPIC (complete down to $K p \sim 19$ mag; Huber et al. 2016) within 4 arcsec (approximately the Kepler pixel size). This last criterion removes $<1 \%$ of the FGK stars in our proposed samples, improves catalog reliability by reducing false positives, and simplifies subsequent vetting and Doppler follow-up.

We draw our late-type ( $\mathrm{K}$ and $\mathrm{M}$ dwarf) stellar sample primarily from the SUPERBLINK proper motion database (SB, Lépine \& Shara 2005) and the PanSTARRS-1 survey (PS1, Kaiser 2002). We use a combination of reduced proper motion, optical/NIR color cuts, and/or SED fitting to capture the majority of $\mathrm{M}$ dwarfs $(>85 \%)$ within $100 \mathrm{pc}$ with little contamination from distant giants. In some $K 2$ campaigns we supplement our initial database using SDSS, PS1, and/or other photometry to identify additional targets with smaller proper motions (following Aller et al. 2013). We estimate approximate spectral types (SpTs) using tabulated photometric relations (Kraus \& Hillenbrand 2007; Pecaut \& Mamajek 2013; Rodriguez et al. 2013) and convert SpTs into stellar radii $\left(R_{*}\right)$ based on interferometric studies (Boyajian et al. 2012). Our exact selection criteria for $\mathrm{K}$ and $\mathrm{M}$ dwarfs have evolved with time, but we typically prioritize this low-temperature stellar sample by requiring a signal-to-noise ratio $(\mathrm{S} / \mathrm{N}) \gtrsim 8$ for a single transit of an Earth-sized planet, assuming the demonstrated photometric precision of $K 2$. We additionally set a magnitude limit of $K p<16.5 \mathrm{mag}$ on this late-type dwarf sample to allow feasible spectroscopic characterization.

\subsection{Time-series Photometry}

Our team's photometric pipeline (described by, e.g., Crossfield et al. 2015; Petigura et al. 2015 builds on the approach originally outlined by Vanderburg \& Johnson (2014). We extract time-series photometry from the target pixel files provided by the project using circular, stationary, soft-edged apertures. During $K 2$ operations, solar radiation pressure

\footnotetext{
$\overline{37}$ K2 Programs 79, 120, 1002, 1036, 2104, 2106, 2107, 3104, 3106, 3107, 4011, 4033 .
} 
torques the spacecraft, causing it to roll around the boresight. This motion causes a typical target star to drift across the CCD by $\sim 1$ pixel every $\sim 6 \mathrm{hr}$. This motion of stars across the CCD, when combined with inter- and intra-pixel sensitivity variations and aperture losses, results in significant changes in our aperture photometry.

We remove these stellar brightness variations that correlate with spacecraft orientation by first solving for the roll angle between each frame and an arbitrary reference frame using roughly 100 stars of $K p \sim 12 \mathrm{mag}$ on an arbitrary output channel. Then, we model the time- and roll-dependent brightness variations using a Gaussian process with a squared-exponential kernel. We apply apertures with radii ranging from 1 to 7 pixels and select the aperture that minimizes the residual noise in the corrected light curve (computed on three-hour timescales). This minimization balances two competing effects: larger apertures yield smaller systematic errors (because aperture losses are smaller), while smaller apertures incur less background noise. All of our processed light curves are available for download at the NExScI ExoFOP website. ${ }^{38}$

\subsection{Identifying Transit-like Signals}

We search our calibrated photometry for planetary transits using the TERRA algorithm (Petigura et al. 2013a). After running TERRA, we flag stars with putative transits having $\mathrm{S} / \mathrm{N}>12$ as threshold-crossing events (TCEs) for visual inspection. Below this level, transit signals surely persist but TCEs become dominated by spurious detections. Residual outliers in our photometry prevent us from identifying large numbers of candidates at lower $\mathrm{S} / \mathrm{N}$. In order to reduce the number of spurious detections we require that TCEs have orbital periods $P \geqslant 1$ days, and that they also show three transits. This last criterion sets an upper bound to the longest period detectable in our survey at half the campaign baseline, or $\sim 37$ days. ${ }^{39}$ Thus many longer-period planets likely remain to be found in these data sets, in a manner analogous to the discovery of HIP-116454b in K2's initial engineering run (Vanderburg et al. 2016) and additional single-transit candidates identified in Campaigns 1-3 (Osborn et al. 2016).

In our analysis, each campaign yields roughly 1000 TCEs. The distribution of their orbital periods, shown in Figure 1, reveals discrete peaks at $P=1.5,2,4,8$, and 16 days. These sharp peaks likely correspond to the $6 \mathrm{hr}$ periodicity of smallscale maneuvering tweaks to rebalance solar pressure and/or to the $48 \mathrm{hr}$ periodicity of $K 2$ 's reaction wheel momentum dumps (Van Cleve et al. 2016). Both these effects could induce correlated photometric jitter on integer multiples of this timescale. We also see a smoother increase in TCEs toward longer periods $(P \gtrsim 16$ days) that our manual vetting (described below) shows as corresponding to an increasing false positive rate (FPR) for TCEs showing just 3-5 transit-like events.

In each campaign, our manual vetting process begins with these TCEs and results in well-defined lists of astrophysical variables, including robust planet candidates for further followup and validation. TERRA produces a set of diagnostics for every TCE with a detection above our $\mathrm{S} / \mathrm{N}$ limit, which we use to determine whether the event was likely caused by a

\footnotetext{
38 https://exofop.ipac.caltech.edu

39 The handful of candidates with $P>37$ days were found by visual inspection.
}

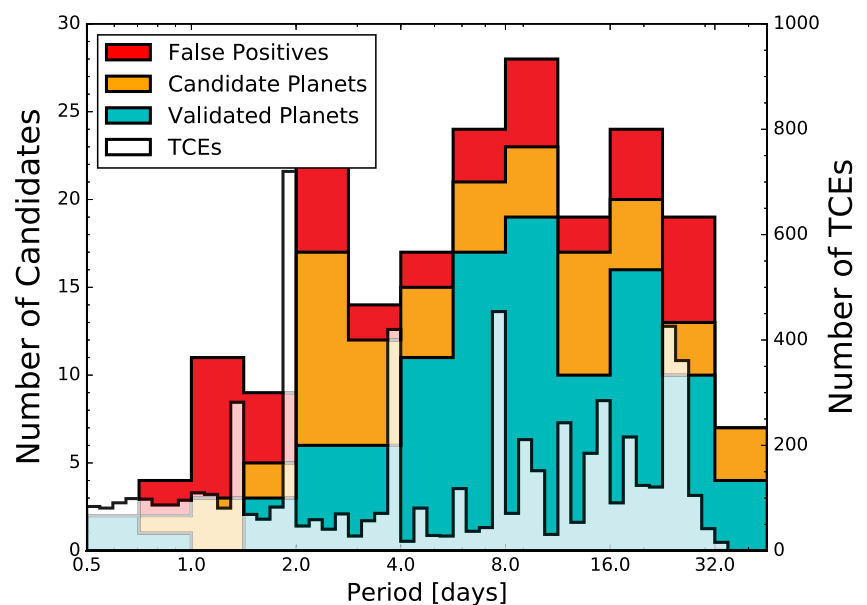

Figure 1. Distribution of orbital periods of transit-like signals identified in our analysis. The pale, narrow-binned histogram (axis at right) indicates the Threshold-Crossing Events (TCEs) identified by TERRA in our initial transit search (see Section 2). The coarser histograms (axis at left) indicate the cumulative distributions of 104 validated planets (blue-green; FPP $<0.01$ ), 30 false positive systems (red; FPP $>0.99$ ), and 63 candidates of indeterminate status (orange).

candidate planet, eclipsing binary, periodic variable, or noise. The diagnostics include a summary of basic fit parameters and a suite of diagnostic plots to visualize the nature of the TCE. These plots include the TERRA periodogram, a normalized phase-folded light curve with a best-fit model, the light curve phased to $180^{\circ}$ to look for eclipses or misidentified periods, the most probable secondary eclipse identified at any phase, and an autocorrelation function. When vetting, the user flags each TCE as an object of interest or not, where objects of interest can be either candidate planets, eclipsing binaries, or variable stars. We elevate any TCE showing no obvious warning signs to the status of "planet candidate," i.e., an event that is almost surely astrophysical in nature, possibly a transiting planet, and not obviously a false positive scenario like a background eclipsing binary. We quantify the FPPs of all our candidates in Section 6. Figure 2 shows an example of a TERRA-derived light curve for a typical candidate.

Once we identify a candidate, we re-run TERRA to search for additional planets in that system as described by Sinukoff et al. (2016). In brief, we mask out the photometry associated with transits of the previously identified candidate and run TERRA again to look for additional box-shaped signals. We repeat this process until no candidates are identified with $\mathrm{S} / \mathrm{N}>8$ or the number of candidates exceeds five. We typically find $<10$ multi-candidate systems per campaign, with a maximum of four planets detected per star.

\section{SUPPORTING OBSERVATIONS}

\subsection{High-resolution Spectroscopy: Observations}

\subsubsection{Keck/HIRES}

We obtained high-resolution optical spectra of 83 planet candidate hosts using the HIRES echelle spectrometer (Vogt 1994) on the $10 \mathrm{~m}$ Keck I telescope. These spectra were collected using the standard procedures of the California Planet Search (CPS; Howard et al. 2010). We used the "C2" decker $\left(0 . \prime 87 \times 14^{\prime \prime}\right.$ slit $)$, which is long enough to simultaneously measure the spectra of the target star and the sky background 

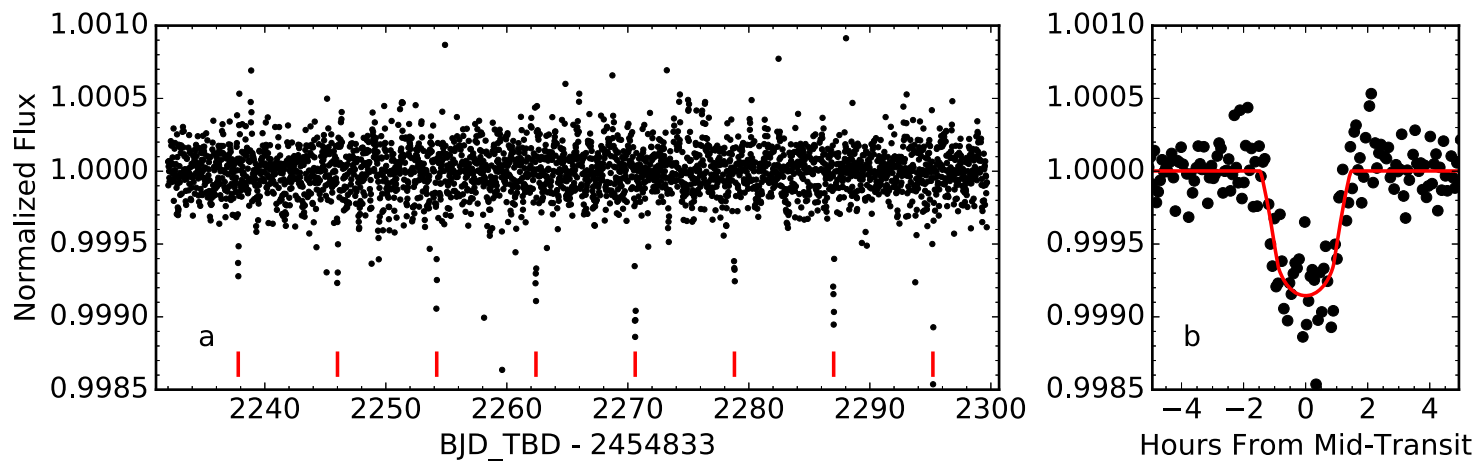

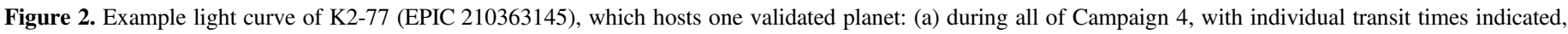
and (b) phase-folded, with the best-fit transit model overplotted in red. The transit parameters for all candidates are listed in Table 8.

with spectral resolution $R=55,000$. The sky was subtracted from each stellar spectrum. We used the HIRES exposure meter to automatically terminate each exposure once the desired $\mathrm{S} / \mathrm{N}$ was reached, typically after 1-20 minutes. For stars with $V<13.0$ mag, exposure levels were set to achieve $\mathrm{S} / \mathrm{N}=45$ per pixel at $550 \mathrm{~nm}$. Exposures of fainter stars were terminated at $\mathrm{S} / \mathrm{N}=32$ per pixel-enough to derive stellar parameters while keeping exposure times reasonable. For stars that were part of subsequent Doppler campaigns, we measured additional HIRES spectra with higher S/N. These RV measurements will be the subject of a series of forthcoming papers.

\subsubsection{Automated Planet Finder (APF)/Levy}

We obtained spectra of 27 candidate host stars using the Levy high-resolution optical spectrograph mounted at the APF. Each spectrum covers a continuous wavelength range from 374 to $970 \mathrm{~nm}$. We observed the stars using either the $2^{\prime \prime} \times 8^{\prime \prime}$ slit for a spectral resolution of $R \approx 80,000$, or, to minimize sky contamination, the $1^{\prime \prime} \times 3^{\prime \prime}$ slit for a spectral resolution of $R \approx 100,000$. We initially observed all bright targets using the $2^{\prime \prime} \times 8^{\prime \prime}$ slit to maximize $\mathrm{S} / \mathrm{N}$ but soon noticed that sky contamination was a serious problem on nights with a full or gibbous moon. All APF spectra collected after 2015 May 21 were observed using the $1^{\prime \prime} \times 3^{\prime \prime}$ decker. In all cases, we collected three consecutive exposures and combined the extracted 1D spectra using a sigma-clipped mean to reject cosmic rays. All targets were observed at just a single epoch. The final $\mathrm{S} / \mathrm{N}$ of the combined spectra ranges from roughly 25 to 50 per pixel.

\subsubsection{MPG $2.2 \mathrm{~m} / \mathrm{FEROS}$}

We obtained spectra of a small number of candidate stellar hosts using the FEROS fiber-fed echelle spectrograph (Kaufer \& Pasquini 1998) at the $2.2 \mathrm{~m}$ MPG telescope. Each spectrum covers a continuous wavelength range from 350 to $920 \mathrm{~nm}$ with an average resolution of $R \sim 48,000$. Our FEROS exposure times were chosen according to the brightness of each target and ranged from 10 to 30 minutes. Simultaneously with the science images we acquired spectra of a ThAr lamp for wavelength calibration.

The FEROS data are processed through a dedicated pipeline built from a modular code (CERES, R. Brahm et al. 2016, in preparation) designed to reduce, extract and analyze data from different echelle spectrographs in an automated, homogeneous and robust way. This pipeline is similar to the calibration and optimal extraction approach described by Jordán et al. (2014).
We compute a global wavelength solution from the calibration ThAr image by fitting a Chebyshev polynomial as function of the pixel position and echelle order number. The instrumental velocity drifts during the night are computed using the the extracted spectra of the ThAr lamp acquired during the science observations with the reference fiber. The barycentric correction is performed using the JPLephem package. Radial velocities (RVs) and bisector spans are determined by crosscorrelating the continuum-normalized stellar spectrum with a binary mask derived from a G2 dwarf's spectrum (for more details see, e.g., Baranne et al. 1979; Queloz 1995). We normalize the stellar continuum to minimize the systematic errors that would be induced in the derived velocity by differences in spectral slope caused by different reddening or stellar type.

\subsection{High-resolution Spectroscopy: Methods and Results}

As part of our false positive analysis (described in Section 6), we use our high-resolution Keck/HIRES spectra to search for additional spectral lines in the stellar spectra. This method is sensitive to secondary stars that lie within 0 " 4 of the primary star (one half of the slit width) and that are as faint as $1 \%$ of the apparent brightness of the primary star (Kolbl et al. 2015). The approach therefore complements the $\mathrm{AO}$ and speckle imaging described in Section 3.3 (Ciardi et al. 2015; Teske et al. 2015).

The search for secondary lines in the HIRES spectra begins with a match of the primary spectrum to a catalog of nearby, slowly rotating, FGKM stars from the CPS. The best match from the catalog is identified, subtracted from the primary spectrum, and the residuals are then searched (using the same catalog) to identify any fainter second spectrum. This method is insensitive to companion stars with velocity offsets of $\lesssim 10 \mathrm{~km} \mathrm{~s}^{-1}$, in which cases multiple stellar lines would be blended too closely together. This method is optimized for slowly rotating FGKM stars, so stars earlier than F and those with $v \sin i>10 \mathrm{~km} \mathrm{~s}^{-1}$ are more difficult to detect due to their having fewer and/or broader spectral lines. The technique is less sensitive for stars with $T_{\text {eff }} \lesssim 3500 \mathrm{~K}$ due to the small number of such stars in the CPS catalog. The derived constraints for all targets are listed in Table 3, and we use them in our false positive analysis described in Section 6. Figure 3 shows an example of a Keck/HIRES spectrum, together with the secondary line search results and derived stellar parameters (see Section 4).

We performed a similar analysis for the subset of stars observed by the FEROS spectrograph. Table 1 lists these stars, 

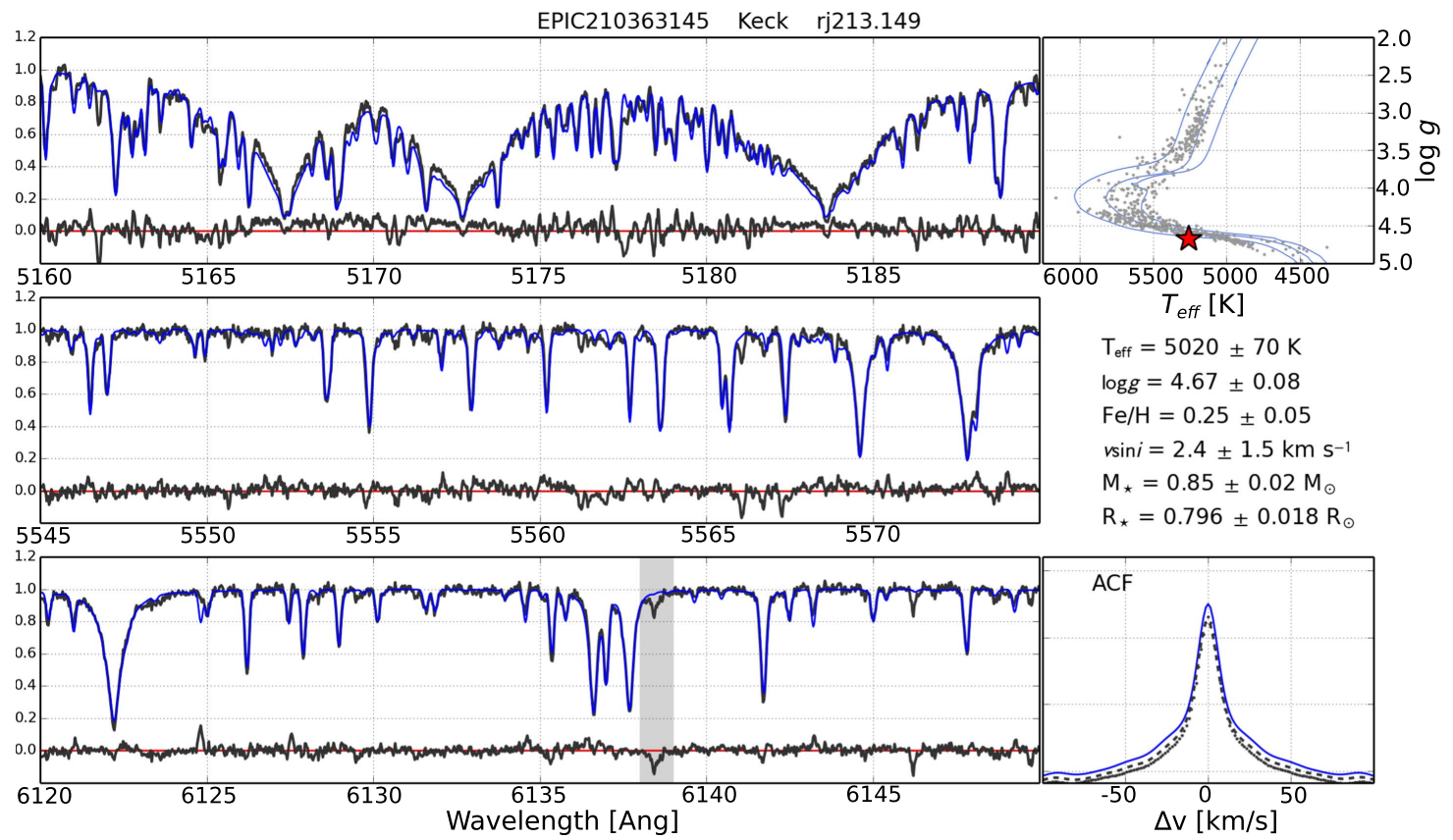

Figure 3. Example Keck/HIRES stellar spectrum (blue), template match (black), and derived parameters for K2-77 (EPIC 210363145). The star has low $v$ sin $i$, moderate $T_{\text {eff }}$, and shows no evidence for additional stellar companions in the spectroscopic autocorrelation function (ACF). The upper-right panel plots the derived stellar parameters against the parameters of the SpecMatch template stars. Stellar parameters for all targets are listed in Table 7, and results of ACF analyses are in Table 3 .

Table 1

FEROS Follow-up Observations

\begin{tabular}{|c|c|}
\hline$\overline{\mathrm{EPIC}}$ & Observation Note \\
\hline 201176672 & Multiple peaks in CCF; likely stellar blend. \\
\hline 201270176 & Multiple peaks in CCF; likely stellar blend. \\
\hline 202088212 & Multiple peaks in CCF; likely stellar blend. \\
\hline 203929178 & Multiple peaks in CCF; likely stellar blend. \\
\hline 204873331 & Multiple peaks in CCF; likely stellar blend. \\
\hline 203485624 & Very broad CCF peak, $v \sin i>50 \mathrm{~km} \mathrm{~s}^{-1}$. \\
\hline 205148699 & Single-peaked CCF, phased RV variations of $\pm 28 \mathrm{~km} \mathrm{~s}^{-1}$. \\
\hline 201626686 & Single-peaked CCF, unphased RV jitter of $\pm 50 \mathrm{~m} \mathrm{~s}^{-1}$. \\
\hline 203771098 & Single-peaked CCF, RV variations $<20 \mathrm{~m} \mathrm{~s}^{-1}$. \\
\hline 201505350 & $\begin{array}{l}\text { Single-peaked CCF, } \sim 20 \mathrm{~m} \mathrm{~s}^{-1} \mathrm{RV} \text { variation between two } \\
\text { epochs. }\end{array}$ \\
\hline 201862715 & Single-peaked CCF. \\
\hline
\end{tabular}

most of which host candidate hot Jupiters. Three show obvious signs of multiple peaks in the stellar cross-correlation, indicating these sources are blends of multiple stars; a fourth shows an extremely high rotational velocity. As described in Section 6, we find FPPs of $>50 \%$ for all four of these systems, indicating that most are likely false positives and low-priority targets for future follow-up.

By obtaining FEROS spectra at multiple epochs, we detect RV variations from EPIC 205148699 in phase with the transit signal and with semi-amplitude $K \sim 28 \mathrm{~km} \mathrm{~s}^{-1}$, indicating that this system is an eclipsing stellar binary. For EPIC 201626686, $11 \mathrm{RV}$ measurements over 40 days reveal variations at the level of $\pm 50 \mathrm{~m} \mathrm{~s}^{-1}$. Since these variations are not in phase with the orbital period of the detected transits, we do not consider this system to be a false positive. Finally, multiple RV measurements also set as an upper limit on the RV variations of K2-24 (EPIC 203771098) of $<20 \mathrm{~m} \mathrm{~s}^{-1}$ (consistent with the analysis of Petigura et al. 2016). Our analysis in Section 6 ultimately
Table 2

False Positive Rates

\begin{tabular}{ll}
\hline \hline Category & FP Rate \\
\hline$R_{P} \leqslant 2 R_{\oplus}$ & 0.07 \\
$2 \leqslant R_{P} / R_{\oplus} \leqslant 8$ & 0.08 \\
$R_{P} \geqslant 8 R_{\oplus}$ & 0.54 \\
$P \leqslant 3$ days & 0.36 \\
$3 \leqslant P \leqslant 15$ & 0.12 \\
$P \geqslant 15$ days & 0.21 \\
Entire Sample & 0.20 \\
\hline
\end{tabular}

finds FPP $<0.01$ for all three of these systems, indicating that these are validated planets.

Single-epoch FEROS observations reveal that both K2-19 (EPIC 201505350) and EPIC 201862715 are single-lined dwarf stars, consistent with our validation of the former (the latter has a close stellar companion that prevents us from validating the system; see Section 6). A second observation of K2-19 taken three days later shows an RV variation of $\sim 20 \mathrm{~m} \mathrm{~s}^{-1}$, roughly consistent with the RV signal reported by Barros et al. (2015).

\subsection{High-resolution Imaging}

\subsubsection{Observations}

We obtained HRI for 164 of our candidate systems. Our primary instrument for this work was NIRC2 at the $10 \mathrm{~m}$ Keck II telescope, with which we observed 110 systems. Most were observed in Natural Guide Star (NGS) mode, though we used Laser Guide Star (LGS) mode for a subset of targets orbiting fainter stars. As part of multi-semester program GN2015B-LP-5 (PI Crossfield) at Gemini Observatory, we observed 40 systems with the NIRI camera (Hodapp 2003) in the $K$-band using NGS or LGS modes. We also observed 33 stars with PHARO/PALM-3000 (Hayward et al. 2001; Dekany 
Table 3

HIRES Follow-up Observations

\begin{tabular}{|c|c|c|}
\hline EPIC & Flag & Note \\
\hline 201295312 & 1 & $1 \%$ \\
\hline 201338508 & 1 & $1 \%$ \\
\hline 201367065 & 1 & $1 \%$ \\
\hline 201384232 & 1 & $1 \%$ \\
\hline 201393098 & 1 & $1 \%$ \\
\hline 201403446 & 1 & $1 \%$ \\
\hline 201445392 & 1 & $1 \%$ \\
\hline 201465501 & 1 & $1 \%$ \\
\hline 201505350 & 1 & $1 \%$ \\
\hline 201546283 & 1 & $1 \%$ \\
\hline 201549860 & 1 & $1 \%$ \\
\hline 201577035 & 1 & $1 \%$ \\
\hline 201613023 & 1 & $1 \%$ \\
\hline 201629650 & 1 & $1 \%$ \\
\hline 201647718 & 1 & $1 \%$ \\
\hline 201677835 & 1 & $1 \%$ \\
\hline 201702477 & 1 & $1 \%$ \\
\hline 201713348 & 1 & $1 \%$ \\
\hline 201736247 & 1 & $1 \%$ \\
\hline 201754305 & 1 & $1 \%$ \\
\hline 201828749 & 1 & $1 \%$ \\
\hline 201912552 & 1 & $1 \%$ \\
\hline 201920032 & 1 & $1 \%$ \\
\hline 202071401 & 1 & $1 \%$ \\
\hline 202083828 & 1 & $1 \%$ \\
\hline 202089657 & 1 & $1 \%$ \\
\hline 202675839 & 1 & $1 \%$ \\
\hline 203771098 & 1 & $1 \%$ \\
\hline 203826436 & 1 & $1 \%$ \\
\hline 204129699 & 1 & $1 \%$ \\
\hline 204221263 & 1 & $1 \%$ \\
\hline 204890128 & 1 & $1 \%$ \\
\hline 205071984 & 1 & $1 \%$ \\
\hline 205570849 & 1 & $1 \%$ \\
\hline 205916793 & 1 & $1 \%$ \\
\hline 205924614 & 1 & $1 \%$ \\
\hline 205944181 & 1 & $1 \%$ \\
\hline 205999468 & 1 & $1 \%$ \\
\hline 206011496 & 1 & $1 \%$ \\
\hline 206011691 & 1 & $1 \%$ \\
\hline 206024342 & 1 & $1 \%$ \\
\hline 206026136 & 1 & $1 \%$ \\
\hline 206026904 & 1 & $1 \%$ \\
\hline 206036749 & 1 & $1 \%$ \\
\hline 206038483 & 1 & $1 \%$ \\
\hline 206044803 & 1 & $1 \%$ \\
\hline 206061524 & 1 & $1 \%$ \\
\hline 206096602 & 1 & $1 \%$ \\
\hline 206101302 & 1 & $1 \%$ \\
\hline 206125618 & 1 & $1 \%$ \\
\hline 206144956 & 1 & $1 \%$ \\
\hline 206153219 & 1 & $1 \%$ \\
\hline 206154641 & 1 & $1 \%$ \\
\hline 206155547 & 1 & $1 \%$ \\
\hline 206159027 & 1 & $1 \%$ \\
\hline 206181769 & 1 & $1 \%$ \\
\hline 206192335 & 1 & $1 \%$ \\
\hline 206245553 & 1 & $1 \%$ \\
\hline 206247743 & 1 & $1 \%$ \\
\hline 206268299 & 1 & $1 \%$ \\
\hline 206348688 & 1 & $1 \%$ \\
\hline 206432863 & 1 & $1 \%$ \\
\hline 206439513 & 1 & $1 \%$ \\
\hline 206495851 & 1 & $1 \%$ \\
\hline
\end{tabular}

Table 3

(Continued)

\begin{tabular}{|c|c|c|}
\hline EPIC & Flag & Note \\
\hline 210363145 & 1 & $1 \%$ \\
\hline 210389383 & 1 & $1 \%$ \\
\hline 210400751 & 1 & $1 \%$ \\
\hline 210402237 & 1 & $1 \%$ \\
\hline 210403955 & 1 & $1 \%$ \\
\hline 210414957 & 1 & $1 \%$ \\
\hline 210448987 & 1 & $1 \%$ \\
\hline 210483889 & 1 & $1 \%$ \\
\hline 210484192 & 1 & $1 \%$ \\
\hline 210508766 & 1 & $1 \%$ \\
\hline 210577548 & 1 & $1 \%$ \\
\hline 210609658 & 1 & $1 \%$ \\
\hline 210666756 & 1 & $1 \%$ \\
\hline 210707130 & 1 & $1 \%$ \\
\hline 210718708 & 1 & $1 \%$ \\
\hline 210731500 & 1 & $1 \%$ \\
\hline 210754505 & 1 & $1 \%$ \\
\hline 210894022 & 1 & $1 \%$ \\
\hline 210957318 & 1 & $1 \%$ \\
\hline 210968143 & 1 & $1 \%$ \\
\hline 211089792 & 1 & $1 \%$ \\
\hline 211099781 & 1 & $1 \%$ \\
\hline 211152484 & 1 & $1 \%$ \\
\hline 201637175 & 2 & $\mathrm{~N} / \mathrm{A}$; star too cool \\
\hline 203710387 & 2 & $\mathrm{~N} / \mathrm{A}$; star too cool \\
\hline 202126852 & 3 & $\mathrm{~N} / \mathrm{A} ;$ high $v \sin i$ \\
\hline 202126888 & 3 & $\mathrm{~N} / \mathrm{A} ;$ high $v \sin i$ \\
\hline 205703094 & 3 & $\mathrm{~N} / \mathrm{A}$; Vsini too high \\
\hline 208833261 & 3 & $\mathrm{~N} / \mathrm{A} ;$ high $v \sin i$ \\
\hline 210954046 & 3 & $\mathrm{~N} / \mathrm{A} ;$ high $v \sin i$ \\
\hline 210958990 & 3 & $\mathrm{~N} / \mathrm{A} ;$ high $v \sin i$ \\
\hline 211147528 & 3 & $\mathrm{~N} / \mathrm{A} ;$ high $v \sin i$ \\
\hline 206027655 & 4 & Marginal detection of $5 \%$ binary at $-10 \mathrm{~km} \mathrm{~s}^{-1}$. \\
\hline 206028176 & 4 & Marginal detection at $10 \mathrm{~km} \mathrm{~s}^{-1}$ separation \\
\hline 201324549 & 5 & triple star system; $\sim 20 \%$ brightness \\
\hline 202088212 & 5 & companion; $3-13 \%$ as bright as primary; $25 \mathrm{~km} \mathrm{~s}^{-1}$ \\
\hline 203753577 & 5 & $15 \%$ companion at $16 \mathrm{~km} \mathrm{~s}^{-1}$ separation \\
\hline 205947161 & 5 & Nearly equal flux binary \\
\hline 206135267 & 5 & Obvious Binary; $50 \%$ flux of primary \\
\hline 206267115 & 5 & $\mathrm{SB} 2$; near equal at $80 \mathrm{~km} \mathrm{~s}^{-1}$ \\
\hline 206543223 & 5 & SB2; $23 \%$ flux of primary \\
\hline 209036259 & 5 & Obvious triple system. \\
\hline 210401157 & 5 & Strange, composite spectrum. \\
\hline 210513446 & 5 & $\mathrm{SB} 2 ; 2 \%$ companion at del-RV $=122 \mathrm{~km} \mathrm{~s}^{-1}$ \\
\hline 210558622 & 5 & $\mathrm{SB} 2 ; 3 \%$ companion at del-RV $=119 \mathrm{~km} \mathrm{~s}^{-1}$ \\
\hline 210744674 & 5 & SB2; equal flux secondary \\
\hline 210789323 & 5 & $\mathrm{SB} 2 ; 22 \%$ companion at del-RV $=-83 \mathrm{~km} \mathrm{~s}^{-1}$ \\
\hline 210903662 & 5 & SB2; near equal binary \\
\hline
\end{tabular}

Notes.

${ }^{1}$ No detection of second spectrum at noted flux ratio.

${ }^{2}$ Star is unfit for ReaMatch: $T_{\text {eff }}$ below $3500 \mathrm{~K}$.

${ }^{3}$ Star is unfit for ReaMatch: $v \sin i$ above $10 \mathrm{~km} \mathrm{~s}^{-1}$.

4 Ambiguous detection.

${ }^{5}$ Obvious detection.

(This table is available in machine-readable form.)

et al. 2013) at the $5 \mathrm{~m}$ Hale Telescope and 14 systems with LMIRCam at LBT (Leisenring et al. 2012), all at the $K$-band. We observed 39 stars at visible wavelengths using the automated Robo-AO laser adaptive optics system at the Palomar $1.5 \mathrm{~m}$ telescope (Baranec et al. 2013, 2014). These 
Table 4

FEROS Radial Velocities

\begin{tabular}{|c|c|c|c|c|c|}
\hline EPIC & BJD & $\mathrm{RV}$ & $\sigma_{R V}$ & $t_{\text {int }}$ & $\mathrm{S} / \mathrm{N}$ \\
\hline 203294831 & 2457182.56854355 & 6.235 & 0.093 & 600 & 45 \\
\hline 203771098 & 2457182.57744262 & 0.713 & 0.010 & 600 & 82 \\
\hline 201176672 & 2457182.58920619 & 41.702 & 0.029 & 1800 & 25 \\
\hline 201626686 & 2457182.62251241 & 49.133 & 0.015 & 600 & 49 \\
\hline 203929178 & 2457182.67319105 & -130.200 & 0.221 & 600 & 54 \\
\hline 203771098 & 2457183.50141524 & 0.725 & 0.010 & 600 & 78 \\
\hline 203929178 & 2457183.53736243 & 64.251 & 0.276 & 600 & 51 \\
\hline 201626686 & 2457183.54133676 & 49.090 & 0.011 & 600 & 76 \\
\hline 204873331 & 2457183.74219492 & 48.694 & 0.620 & 900 & 46 \\
\hline 203485624 & 2457183.75453967 & 93.324 & 1.867 & 900 & 47 \\
\hline 203294831 & 2457183.76528655 & 7.172 & 0.076 & 600 & 46 \\
\hline 205148699 & 2457183.77680036 & -41.853 & 0.058 & 900 & 43 \\
\hline 203294831 & 2457184.51873516 & 6.106 & 0.118 & 600 & 34 \\
\hline 204873331 & 2457184.54186421 & -39.425 & 0.559 & 900 & 43 \\
\hline 205148699 & 2457184.55524152 & -32.892 & 0.055 & 900 & 46 \\
\hline 203771098 & 2457184.57256456 & 0.707 & 0.010 & 600 & 90 \\
\hline 203929178 & 2457184.58722675 & -80.916 & 0.226 & 600 & 57 \\
\hline 203485624 & 2457184.70016264 & -8.811 & 1.983 & 900 & 54 \\
\hline 201176672 & 2457185.53558417 & 41.739 & 0.023 & 1800 & 31 \\
\hline 201626686 & 2457185.55 & 49.105 & 0.011 & 600 & 74 \\
\hline 203294831 & 2457185.61 & 6.799 & 0.076 & 600 & 43 \\
\hline 203929178 & 2457185.6 & -3.928 & 0.841 & 600 & 53 \\
\hline 205148699 & 2457185.68276227 & -61.542 & 0.055 & 900 & 45 \\
\hline 204873331 & 2457185.76186667 & -51.492 & 0.320 & 900 & 53 \\
\hline 203485624 & 2457185.77667465 & -58.772 & 3.136 & 900 & 39 \\
\hline 203485624 & 2457186.59017442 & -20.641 & 1.277 & 900 & 40 \\
\hline 205148699 & 2457186.60319043 & -77.486 & 0.068 & 900 & 35 \\
\hline 205148699 & 2457186.61615822 & -77.408 & 0.060 & 900 & 41 \\
\hline 204873331 & 2457186.62889121 & 6.458 & 0.327 & 900 & 35 \\
\hline 203929178 & 2457186.68052468 & -20.963 & 0.161 & 600 & 44 \\
\hline 203294831 & 2457186.80984085 & 5.598 & 0.121 & 600 & 37 \\
\hline 203929178 & 2457187.61 & -76.018 & 0.467 & 600 & 59 \\
\hline 203485624 & 2457 & -121.651 & 2.046 & 900 & 52 \\
\hline 201626686 & 2457190.49169087 & 49.070 & 0.010 & 600 & 80 \\
\hline 203485624 & 2457190.57993074 & -17.006 & 1.498 & 900 & 58 \\
\hline 203294831 & 2457190.59359013 & 5.611 & 0.072 & 600 & 61 \\
\hline 203294831 & 2457191.51831070 & 5.522 & 0.083 & 600 & 50 \\
\hline 203485624 & 2457191.53066007 & 69.097 & 1.920 & 900 & 3 \\
\hline 203929178 & 2457191.54262838 & -88.136 & 0.276 & 600 & 53 \\
\hline 204873331 & 2457191.55366638 & 42.413 & 0.630 & 900 & 36 \\
\hline 201176672 & 2457192.52186285 & 41.755 & 0.026 & 1800 & 26 \\
\hline 201626686 & 2457192.54283939 & 49.094 & 0.015 & 600 & 46 \\
\hline 203294831 & 2457192.56384913 & 1.127 & 0.770 & 600 & 21 \\
\hline 201626686 & 24571 & 49.149 & 0.015 & 600 & 49 \\
\hline 2032 & 24571 & 5.362 & 0.113 & 600 & 36 \\
\hline 203485624 & 2457193.54441500 & -62.799 & 1.150 & 900 & 35 \\
\hline 204873331 & 2457193.56058533 & -39.060 & 0.228 & 900 & 62 \\
\hline 205148699 & 2457193.57490799 & -38.108 & 0.048 & 900 & 54 \\
\hline 203929178 & 2457193.74554128 & -27.479 & 0.915 & 600 & 62 \\
\hline 201176672 & 2457194.51197780 & 41.713 & 0.022 & 1800 & 32 \\
\hline 201626686 & 2457194.52827067 & 49.060 & 0.011 & 600 & 73 \\
\hline 203929178 & 2457194.54604512 & -78.496 & 0.263 & 600 & 63 \\
\hline 203485624 & 2457194.57421092 & 40.485 & 2.366 & 900 & 40 \\
\hline 203294831 & 2457194.58577034 & 2.920 & 0.183 & 600 & 50 \\
\hline 203771098 & 2457194.59578556 & 0.720 & 0.010 & 600 & 97 \\
\hline 205148699 & 2457194.60718775 & -65.417 & 0.047 & 900 & 55 \\
\hline 204873331 & 2457194.76473872 & -30.284 & 0.301 & 900 & 42 \\
\hline 203485624 & 2457195.57768234 & -30.056 & 3.353 & 900 & 43 \\
\hline 203929178 & 2457195.59152469 & 62.791 & 0.432 & 600 & 40 \\
\hline 204873331 & 2457195.60248537 & 51.545 & 0.467 & 900 & 41 \\
\hline 205148699 & 2457195.61518621 & -78.246 & 0.073 & 900 & 33 \\
\hline 203294831 & 2457195.67227197 & 2.509 & 0.482 & 600 & 21 \\
\hline 203771098 & 2457195.68202812 & 0.683 & 0.015 & 600 & 32 \\
\hline 294831 & 2457211.65688105 & 4.917 & 0.081 & 600 & 43 \\
\hline
\end{tabular}

Table 4

(Continued)

\begin{tabular}{lcrcrc}
\hline \hline EPIC & BJD & \multicolumn{1}{c}{$\mathrm{RV}$} & $\sigma_{R V}$ & \multicolumn{1}{c}{$t_{\text {int }}$} & $\mathrm{S} / \mathrm{N}$ \\
\hline 203929178 & 2457211.67035362 & 74.751 & 0.344 & 600 & 55 \\
203771098 & 2457211.68015495 & 0.722 & 0.010 & 600 & 95 \\
203485624 & 2457211.69331295 & 42.800 & 6.707 & 900 & 41 \\
204873331 & 2457211.70909075 & 0.643 & 0.274 & 900 & 54 \\
205148699 & 2457211.72234337 & -56.025 & 0.047 & 900 & 53 \\
201626686 & 2457218.47908419 & 49.071 & 0.012 & 900 & 60 \\
201626686 & 2457219.46201643 & 49.100 & 0.013 & 600 & 57 \\
201626686 & 2457220.48312511 & 49.054 & 0.013 & 600 & 57 \\
201626686 & 2457221.48191204 & 49.087 & 0.012 & 600 & 69 \\
202088212 & 2457408.67412585 & -17.112 & 0.021 & 900 & 87 \\
201505350 & 2457409.82308403 & 7.334 & 0.011 & 1500 & 45 \\
201270176 & 2457410.85912543 & 91.924 & 0.088 & 1100 & 37 \\
201862715 & 2457410.87002659 & 13.448 & 0.010 & 420 & 69 \\
201505350 & 2457412.79619955 & 7.369 & 0.011 & 1500 & 47 \\
201862715 & 2457412.84747439 & 13.645 & 0.010 & 420 & 75 \\
201270176 & 2457413.72138662 & 80.691 & 0.064 & 1100 & 52 \\
\hline
\end{tabular}

(This table is available in machine-readable form.)

data were acquired and reduced separately using the standard Robo-AO procedures outlined by Law et al. (2014).

We acquired the data from all our large-aperture AO observations (NIRC2, NIRI, LMIRCam, PHARO) in a consistent manner. We observed at up to nine dither positions, using integration times short enough to avoid saturation (typically $\leqslant 60 \mathrm{~s}$ ). We use the dithered images to remove sky background and dark current, and then align, flat-field, and stack the individual images.

Through our Long-Term Gemini program we also acquired high-resolution speckle imaging of 32 systems in narrowband filters centered at 692 and $880 \mathrm{~nm}$ using the DSSI camera (Horch et al. 2009, 2012) at the Gemini North telescope. The DSSI observing procedure is typically to center the target star in the field, set up guiding, and take data using $60 \mathrm{~ms}$ exposures. The total integration time varies by target brightness and observing conditions. We measure background sensitivity in a series of concentric annuli around the target star. The innermost data point represents the telescope diffraction limit, within which we set our sensitivity to zero. After measuring our sensitivity across the DSSI field of view, we interpolate through the measurements using a cubic spline to produce a smooth sensitivity curve.

\subsubsection{Contrast and Stellar Companions}

We estimate the sensitivity of all our HRI data by injecting fake sources into the final combined images with separations at integral multiples of the central source's FWHM (see, e.g., Adams et al. 2012; Ziegler et al. 2016). Figure 4 shows an example of a Keck/NIRC2 NGS image and the resulting $5 \sigma$ contrast curve. The median contrast curves achieved by each HRI instrument are shown in Figure 5 together with all detected stellar companions. The companions are also listed in Table 5. Contrast curves for each individual system are uploaded to the ExoFOP website. In addition, Table 10 includes the total integration times and filters used for all candidates observed in our follow-up efforts.

The contrast curves are plotted in the band of observations, which ranges from optical wavelengths (DSSI; Robo-AO) to the $K$-band (large-aperture AO systems). These in-band 
Table 5

HRI-detected Stellar Companions

\begin{tabular}{|c|c|c|c|c|c|}
\hline EPIC & $\begin{array}{l}r_{a p} \\
\left({ }^{\prime \prime}\right)\end{array}$ & $\begin{array}{c}\rho \\
\left({ }^{\prime \prime}\right)\end{array}$ & $\begin{array}{l}\text { Delta mag } \\
\text { (mag) }\end{array}$ & Filter & Telescope \\
\hline 201176672 & 11.94 & 0.340 & 2.77 & $\mathrm{~K}$ & Keck2 \\
\hline 201295312 & 11.94 & 8.110 & 4.00 & $\mathrm{~K}$ & Keck2 \\
\hline 201295312 & 11.94 & 8.070 & 4.10 & $\mathrm{~K}$ & Palomar \\
\hline 201324549 & 11.94 & 0.090 & 0.43 & $\mathrm{~K}$ & GemN-NIRI \\
\hline 201488365 & $\cdots$ & 4.100 & 9.56 & $\mathrm{i}$ & Robo-AO \\
\hline 201505350 & 11.94 & 0.160 & 0.36 & $\mathrm{~K}$ & Palomar \\
\hline 201546283 & 7.96 & 3.030 & 5.83 & $\mathrm{i}$ & Robo-AO \\
\hline 201546283 & 7.96 & 2.960 & 3.74 & $\mathrm{~K}$ & Keck2 \\
\hline 201626686 & 11.94 & 15.390 & 2.43 & $\mathrm{~K}$ & Palomar \\
\hline 201629650 & 11.94 & 3.210 & 5.79 & $\mathrm{~K}$ & Keck2 \\
\hline 201828749 & 11.94 & 2.540 & 1.97 & $\mathrm{i}$ & Robo-AO \\
\hline 201828749 & 11.94 & 2.450 & 1.07 & $\mathrm{~K}$ & Keck2 \\
\hline 201828749 & 11.94 & 2.440 & 1.11 & $\mathrm{~K}$ & Palomar \\
\hline 201862715 & 11.94 & 1.450 & 0.90 & $\mathrm{i}$ & Robo-AO \\
\hline 201862715 & 11.94 & 1.470 & 0.51 & $\mathrm{~K}$ & Palomar \\
\hline 202059377 & 11.94 & 0.390 & 0.34 & $\mathrm{i}$ & Robo-AO \\
\hline 202059377 & 11.94 & 0.360 & 0.32 & $\mathrm{~K}$ & LBT \\
\hline 202066212 & $\cdots$ & 9.120 & 0.43 & $\mathrm{~K}$ & Palomar \\
\hline 202066212 & $\cdots$ & 10.580 & 2.72 & $\mathrm{~K}$ & Palomar \\
\hline 202066537 & 7.96 & 2.280 & 0.69 & $\mathrm{i}$ & Robo-AO \\
\hline 202066537 & 7.96 & 2.290 & 0.58 & $\mathrm{~K}$ & LBT \\
\hline 202071289 & 11.94 & 0.060 & 0.09 & $\mathrm{~K}$ & Keck2 \\
\hline 202071401 & 15.92 & 2.880 & 2.49 & $\mathrm{i}$ & Robo-AO \\
\hline 202071401 & 15.92 & 6.230 & 5.05 & $\mathrm{i}$ & Robo-AO \\
\hline 202071401 & 15.92 & 2.840 & 1.70 & $\mathrm{~K}$ & Keck2 \\
\hline 202071401 & 15.92 & 2.840 & 1.79 & $\mathrm{~K}$ & Palomar \\
\hline 202071401 & 15.92 & 6.050 & 5.23 & $\mathrm{~K}$ & Palomar \\
\hline 202071645 & 11.94 & 3.700 & 7.19 & $\mathrm{i}$ & Robo-AO \\
\hline 202071645 & 11.94 & 3.360 & 7.06 & $\mathrm{~K}$ & Palomar \\
\hline 202071645 & 11.94 & 3.630 & 7.43 & $\mathrm{~K}$ & Palomar \\
\hline 202071645 & 11.94 & 9.290 & 3.67 & $\mathrm{~K}$ & Palomar \\
\hline 202071645 & 11.94 & 10.850 & 5.88 & $\mathrm{~K}$ & Palomar \\
\hline 202083828 & 11.94 & 5.530 & 5.02 & $\mathrm{i}$ & Robo-AO \\
\hline 202088212 & 15.92 & 1.310 & 6.79 & $\mathrm{~K}$ & Keck2 \\
\hline 202089657 & 15.92 & 8.550 & 5.28 & $\mathrm{~K}$ & Palomar \\
\hline 202089657 & 15.92 & 9.210 & 5.58 & $\mathrm{~K}$ & Palomar \\
\hline 202089657 & 15.92 & 11.160 & 4.67 & $\mathrm{~K}$ & Palomar \\
\hline 202089657 & 15.92 & 11.630 & 6.73 & $\mathrm{~K}$ & Palomar \\
\hline 202126849 & 15.92 & 4.610 & 5.70 & $\mathrm{i}$ & Robo-AO \\
\hline 202126852 & 15.92 & 7.150 & 7.44 & $\mathrm{~K}$ & Palomar \\
\hline 202126852 & 15.92 & 3.730 & 6.99 & $\mathrm{~K}$ & Palomar \\
\hline 202126852 & 15.92 & 7.170 & 7.38 & $\mathrm{i}$ & Robo-AO \\
\hline 202126887 & 15.92 & 5.770 & 2.79 & $\mathrm{i}$ & Robo-AO \\
\hline 202126887 & 15.92 & 7.260 & 2.33 & $\mathrm{i}$ & Robo-AO \\
\hline 202126887 & 15.92 & 5.580 & 2.53 & $\mathrm{~K}$ & Keck2 \\
\hline 202126887 & 15.92 & 7.200 & 1.41 & $\mathrm{~K}$ & Keck2 \\
\hline 202126888 & 15.92 & 6.700 & 5.98 & $\mathrm{i}$ & Robo-AO \\
\hline 202565282 & 7.96 & 2.170 & 5.19 & $\mathrm{~K}$ & Keck2 \\
\hline 203929178 & 19.9 & 0.110 & 1.37 & $\mathrm{~K}$ & Keck2 \\
\hline 204043888 & 7.96 & 5.520 & 4.73 & $\mathrm{~K}$ & Keck2 \\
\hline 204489514 & 15.92 & 5.390 & 4.49 & $\mathrm{~K}$ & Keck2 \\
\hline 204890128 & 15.92 & 7.510 & 1.55 & $\mathrm{~K}$ & Keck2 \\
\hline 205029914 & 11.94 & 3.320 & 0.87 & $\mathrm{~K}$ & Keck2 \\
\hline 205064326 & 11.94 & 4.270 & 3.92 & $\mathrm{~K}$ & Keck2 \\
\hline 205148699 & 11.94 & 0.090 & 0.83 & $\mathrm{~K}$ & Keck2 \\
\hline 205686202 & 11.94 & 0.790 & 3.82 & $\mathrm{~K}$ & Keck2 \\
\hline 205703094 & 11.94 & 0.140 & 0.37 & $\mathrm{~K}$ & Keck2 \\
\hline 205916793 & 11.94 & 7.300 & 0.35 & $\mathrm{~K}$ & Palomar \\
\hline 205962680 & 11.94 & 0.480 & 0.27 & $\mathrm{~K}$ & Keck2 \\
\hline 205999468 & 7.96 & 18.500 & 3.27 & $\mathrm{~K}$ & Palomar \\
\hline 206011496 & 7.96 & 0.980 & 2.81 & $\mathrm{~K}$ & Keck2 \\
\hline 206047297 & 11.94 & 9.560 & 5.90 & $\mathrm{~K}$ & Palomar \\
\hline 206061524 & 7.96 & 0.410 & 1.37 & $\mathrm{~K}$ & Palomar \\
\hline
\end{tabular}

Table 5

(Continued)

\begin{tabular}{|c|c|c|c|c|c|}
\hline EPIC & $\begin{array}{l}r_{a p} \\
\left({ }^{\prime \prime}\right)\end{array}$ & $\begin{array}{c}\rho \\
\left({ }^{\prime \prime}\right)\end{array}$ & $\begin{array}{l}\text { Delta mag } \\
\quad(\mathrm{mag})\end{array}$ & Filter & Telescope \\
\hline 206192335 & 11.94 & 2.240 & 6.18 & $\mathrm{~K}$ & GemN-NIRI \\
\hline 206192335 & 11.94 & 2.260 & 6.21 & $\mathrm{~K}$ & Palomar \\
\hline 207389002 & 11.94 & 5.940 & 2.36 & $\mathrm{~K}$ & GemS-GNIRS \\
\hline 207389002 & 11.94 & 5.370 & 2.96 & $\mathrm{~K}$ & GemS-GNIRS \\
\hline 207389002 & 11.94 & 5.910 & 2.29 & $\mathrm{i}$ & Robo-AO \\
\hline 207475103 & 15.92 & 0.100 & 0.12 & $\mathrm{~K}$ & LBT \\
\hline 207475103 & 15.92 & 4.340 & 3.18 & $\mathrm{i}$ & Robo-AO \\
\hline 207475103 & 15.92 & 7.610 & -2.11 & $\mathrm{i}$ & Robo-AO \\
\hline 207475103 & 15.92 & 7.700 & 2.51 & $\mathrm{i}$ & Robo-AO \\
\hline 207517400 & 15.92 & 3.530 & 2.63 & $\mathrm{i}$ & Robo-AO \\
\hline 207517400 & 15.92 & 3.430 & 1.92 & $\mathrm{~K}$ & Palomar \\
\hline 207517400 & 15.92 & 8.320 & 1.35 & $\mathrm{~K}$ & Palomar \\
\hline 207517400 & 15.92 & 10.620 & -2.12 & $\mathrm{~K}$ & Palomar \\
\hline 207739861 & 11.94 & 5.440 & 1.62 & $\mathrm{i}$ & Robo-AO \\
\hline 208445756 & 11.94 & 5.970 & 1.37 & $\mathrm{i}$ & Robo-AO \\
\hline 208445756 & 11.94 & 5.850 & 1.19 & $\mathrm{~K}$ & Palomar \\
\hline 208445756 & 11.94 & 11.290 & 3.06 & $\mathrm{~K}$ & Palomar \\
\hline 208445756 & 11.94 & 13.130 & 3.79 & $\mathrm{~K}$ & Palomar \\
\hline 208445756 & 11.94 & 12.000 & 4.05 & $\mathrm{~K}$ & Palomar \\
\hline 209036259 & 15.92 & 4.000 & 2.96 & $\mathrm{i}$ & Robo-AO \\
\hline 210401157 & 5.572 & 0.500 & 2.47 & $\mathrm{a}$ & GemN-Spk \\
\hline 210401157 & 5.572 & 0.490 & 2.27 & $\mathrm{~b}$ & GemN-Spk \\
\hline 210401157 & 5.572 & 0.470 & 1.67 & $\mathrm{~K}$ & Keck2 \\
\hline 210414957 & 15.92 & 0.790 & 2.41 & $\mathrm{~K}$ & GemN-NIRI \\
\hline 210414957 & 15.92 & 1.020 & 4.95 & $\mathrm{~K}$ & GemN-NIRI \\
\hline 210513446 & 7.96 & 0.240 & 1.26 & $\mathrm{~K}$ & GemN-NIRI \\
\hline 210666756 & 5.572 & 2.360 & 1.30 & $\mathrm{~K}$ & GemN-NIRI \\
\hline 210666756 & 5.572 & 7.850 & 1.28 & $\mathrm{~K}$ & GemN-NIRI \\
\hline 210769880 & 15.92 & 0.780 & 5.39 & $\mathrm{~K}$ & GemN-NIRI \\
\hline 210954046 & 7.96 & 2.930 & 1.45 & $\mathrm{~K}$ & GemN-NIRI \\
\hline 210958990 & 11.94 & 1.740 & 2.52 & $\mathrm{a}$ & GemN-Spk \\
\hline 210958990 & 11.94 & 1.790 & 2.80 & $\mathrm{~b}$ & GemN-Spk \\
\hline 210958990 & 11.94 & 1.820 & 1.71 & $\mathrm{~K}$ & Keck2 \\
\hline 211089792 & 15.92 & 4.240 & 0.89 & $\mathrm{~K}$ & GemN-NIRI \\
\hline 211147528 & 15.92 & 1.330 & 6.75 & $\mathrm{~b}$ & GemN-Spk \\
\hline 211147528 & 15.92 & 1.300 & 5.02 & $\mathrm{~K}$ & Keck2 \\
\hline 203099398 & $\cdots$ & 1.970 & 1.67 & $\mathrm{~K}$ & Keck2 \\
\hline 203867512 & $\ldots$ & 0.453 & 0.61 & $\mathrm{~K}$ & Keck2 \\
\hline 204057095 & $\ldots$ & 0.790 & 2.71 & $\mathrm{~K}$ & Keck2 \\
\hline 204057095 & $\cdots$ & 0.870 & 2.97 & $\mathrm{~K}$ & Keck2 \\
\hline 204750116 & $\cdots$ & 2.980 & 5.91 & $\mathrm{~K}$ & Keck2 \\
\hline
\end{tabular}

(This table is available in machine-readable form.)

magnitude differences set upper limits on the maximum amount of blending possible within the Kepler bandpass. If the companion has the same color as the primary, then the measured $\Delta$ mag is indeed the $\Delta K p$. If the companion is redder, then the $K p$-band flux ratio is even smaller. All detected sources are included in Table 5, even though some lie outside of our photometric apertures. In these cases the detected companion has little or no impact on the transit parameters and FPPs derived below. We discuss such considerations more thoroughly in Section 6.2.

\section{STELLAR PARAMETERS}

Stellar parameters are needed to convert the physical properties measured by our transit photometry into useful planetary parameters such as radius $\left(R_{P}\right)$ and incident 
Table 6

Disposition of Multi-star Candidates

\begin{tabular}{|c|c|c|c|}
\hline EPIC & $\rho<4^{\prime \prime}$ & $F_{2} / F_{1}<\delta^{\prime}$ & Comment \\
\hline 201176672 & True & True & Cannot validate candidate. \\
\hline 201295312 & False & True & Same depth for $r=1^{\prime \prime}$ aperture. \\
\hline 201324549 & True & True & Cannot validate candidate. \\
\hline 201546283 & True & True & Cannot validate candidate. \\
\hline 201626686 & False & True & $\begin{array}{l}\text { Shallower transit with } r=1^{\prime \prime} \text {; } \\
\text { likely FP. }\end{array}$ \\
\hline 201629650 & True & True & Cannot validate candidate. \\
\hline 201828749 & True & True & Cannot validate candidate. \\
\hline 201862715 & True & True & Cannot validate candidate. \\
\hline 202059377 & True & True & Cannot validate candidate. \\
\hline 202066537 & True & True & Cannot validate candidate. \\
\hline 202071289 & True & True & Cannot validate candidate. \\
\hline 202071401 & True & True & Cannot validate candidate. \\
\hline 202071645 & True & False & Secondary star sufficiently faint. \\
\hline 202083828 & False & True & Same depth for $r=1^{\prime \prime}$ aperture. \\
\hline 202088212 & True & False & Secondary star sufficiently faint. \\
\hline 202126849 & False & False & Secondary star sufficiently faint. \\
\hline 202126852 & False & False & Secondary star sufficiently faint. \\
\hline 202126887 & False & True & Deeper transit with $r=1^{\prime \prime}$ aperture. \\
\hline 202126888 & False & True & Same depth for $r=1^{\prime \prime}$ aperture. \\
\hline 202565282 & True & False & Secondary star sufficiently faint. \\
\hline 203929178 & True & True & Cannot validate candidate. \\
\hline 204043888 & False & False & Secondary star sufficiently faint. \\
\hline 204489514 & False & False & Secondary star sufficiently faint. \\
\hline 204890128 & False & True & Same depth for $r=1^{\prime \prime}$ aperture. \\
\hline 205029914 & True & True & Cannot validate candidate. \\
\hline 205064326 & False & True & $\begin{array}{l}\text { Shallower transit with } r=1^{\prime \prime} \text {; } \\
\text { likely FP. }\end{array}$ \\
\hline 205148699 & True & True & Cannot validate candidate. \\
\hline 205686202 & True & True & Cannot validate candidate. \\
\hline 205703094 & True & True & Cannot validate candidate. \\
\hline 205916793 & False & True & Deeper transit with $r=1^{\prime \prime}$ aperture. \\
\hline 205999468 & False & True & Same depth for $r=1^{\prime \prime}$ aperture. \\
\hline 206011496 & True & True & Cannot validate candidate. \\
\hline 206061524 & True & True & Cannot validate candidate. \\
\hline 206192335 & True & True & Cannot validate candidate. \\
\hline 207389002 & False & False & Secondary star sufficiently faint. \\
\hline 207475103 & True & True & Cannot validate candidate. \\
\hline 207517400 & True & True & Cannot validate candidate. \\
\hline 207739861 & False & True & Cannot validate candidate. \\
\hline 208445756 & False & True & Cannot validate candidate. \\
\hline 209036259 & False & True & Cannot validate candidate. \\
\hline 210401157 & True & True & Cannot validate candidate. \\
\hline 210414957 & True & True & Cannot validate candidate. \\
\hline 210513446 & True & True & Cannot validate candidate. \\
\hline 210666756 & True & True & Cannot validate candidate. \\
\hline 210958990 & True & True & Cannot validate candidate. \\
\hline 211089792 & False & True & Same depth for $r=1^{\prime \prime}$ aperture. \\
\hline 211147528 & True & True & Cannot validate candidate. \\
\hline
\end{tabular}

irradiation $\left(S_{\text {inc }}\right)$. We use several complementary techniques to infer stellar parameters for our entire sample.

For all stars with Keck/HIRES and/or APF/Levy spectra, we attempt to estimate effective temperatures, surface gravities, metallicities, and rotational velocities using specMatch (Petigura 2015). SpecMatch fits a high-resolution optical spectrum to an interpolated library of model spectra from Coelho et al. (2005), which closely match the spectra of wellcharacterized stars in this temperature range. Uncertainties on $T_{\text {eff }}, \log g$, and $[\mathrm{Fe} / \mathrm{H}]$ from HIRES spectra are $60 \mathrm{~K}$, 0.08-0.10 dex, and 0.04 dex, respectively (Petigura 2015).

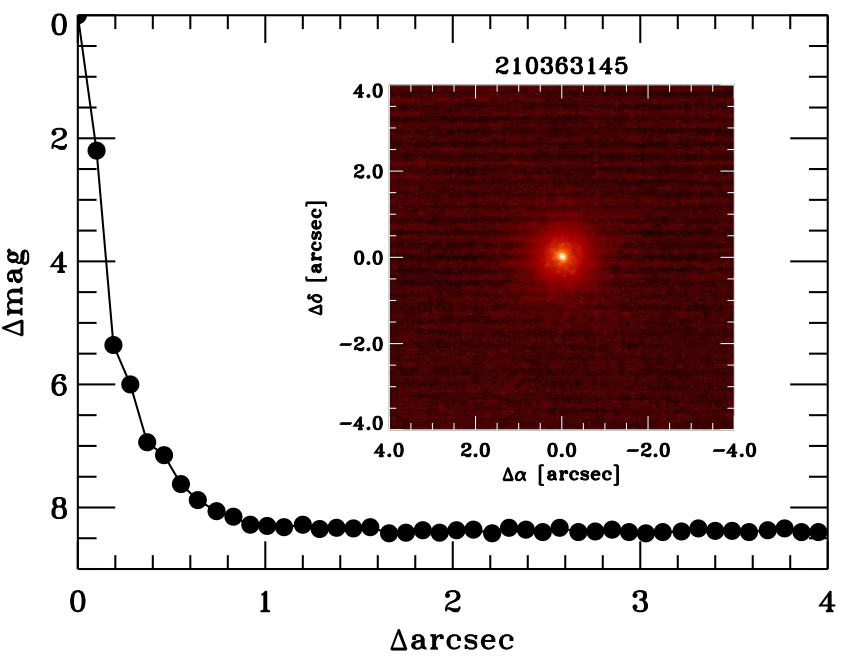

Figure 4. Example constraints on any additional, nearby stars around K2-77 (EPIC 210363145) from Keck/NIRC2 $K$-band adaptive optics imaging. For this target, no companions were detected above the plotted contrast limits. Detected stellar companions around all observed candidates are listed in Table 5 .

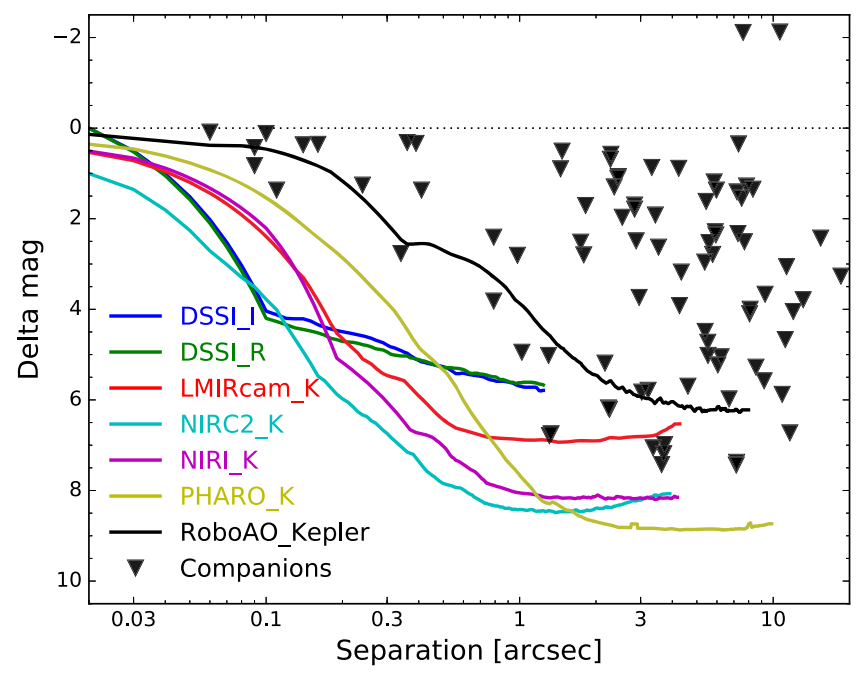

Figure 5. Stellar companions (triangles) detected near our $K 2$ candidate systems and the median contrast achieved with each listed instrument and filter (solid curves). As described in Section 3.3, these detected magnitude differences set upper limits on the maximum amount of blending possible within the Kepler bandpass. Parameters of these nearby stars are listed in Table 5 .

Experience shows that SpecMatch is limited to stars with $T_{\text {eff }} \sim 4700-6500 \mathrm{~K}$ and $v \sin i \lesssim 30 \mathrm{~km} \mathrm{~s}^{-1}$.

The SpecMatch pipeline used to analyze the APF data is identical to the Keck SpecMatch pipeline, except we employ the differential-evolution Markov Chain Monte Carlo (DEMCMC; Ter Braak 2006) fitting engine from ExoPy (Fulton et al. 2013) instead of $\chi^{2}$ minimization. The APF SpecMatch pipeline was empirically calibrated to produce consistent stellar parameters for stars that were observed at both Keck and APF by fitting and subtracting a three-dimensional surface to the residuals of $T_{\text {eff }}, \log g$, and $\mathrm{Fe} / \mathrm{H}$ between the calibrated Keck and initial APF parameters. The errors on the stellar parameters are a quadrature sum of the statistical errors from the DEMCMC fits and the scatter in the APF versus Keck calibration. The scatter in the calibration is generally an order of magnitude 
larger than the statistical errors in the $\mathrm{S} / \mathrm{N}$ regime for the $K 2$ targets observed on APF.

Petigura (2015) assessed the accuracy of SpecMatchderived stellar parameters by modeling the spectra of several samples of touchstone stars with well-measured properties. The properties of these stars were determined from asteroseismology (Huber et al. 2012), detailed LTE spectral modeling, and transit light curve modeling (Torres et al. 2012), and detailed LTE spectral modeling (Valenti \& Fischer 2005). The uncertainties of SpecMatch parameters are dominated by errors in the Coelho et al. (2005) model spectra (e.g., inaccuracies in the line lists, assumption of LTE, etc.). Given that we observe spectra at $\mathrm{S} / \mathrm{N} \gtrsim 35$ per pixel, photon-limited errors are not an appreciable fraction of the overall error budget.

To estimate stellar masses and radii for all stars with SpecMatch parameters, we use the free and open source isochrones Python package (Morton 2015a). This tool accepts as inputs the $T_{\text {eff }}, \log g$, and $[\mathrm{Fe} / \mathrm{H}]$ measured by SpecMatch and interpolates over a grid of stellar models from the Dartmouth Stellar Evolution Database (Dotter et al. 2008). isochrones uses the emcee Markov Chain Monte Carlo package (Foreman-Mackey et al. 2012) to estimate uncertainties, sometimes reporting fractional uncertainties as low as 1\%. Following Sinukoff et al. (2016), we adopt a lower limit of 5\% for the uncertainties on stellar mass and radius to account for the intrinsic uncertainties of the Dartmouth models found by Feiden \& Chaboyer (2012).

Eighty-five stars in our sample lack SpecMatch parameters. For most of these, we adopt the stellar parameters of Huber et al. (2016). This latter analysis relies on the Padova set of stellar models (Marigo et al. 2008), which systematically underestimate the stellar radii of low-mass stars. Follow-up spectroscopy to provide refined parameters for these later-type stars is underway (C. Dressing et al. 2016, in preparation; A. Martinez et al. 2016, in preparation). Our sample includes a small number of stars not considered by Huber et al. (2016), such as targets in K2's Campaign 0. For these, we use isochrones in conjunction with broadband photometry collected from the APASS, 2MASS, and WISE surveys to infer the stellar parameters.

We then use the free, open source LDTk toolkit (Parviainen $\&$ Aigrain 2015) to propagate our measured $T_{\text {eff }}, \log g,[\mathrm{Fe} / \mathrm{H}]$, and their uncertainties into limb-darkening coefficients and associated uncertainties. These limb-darkening parameters act as priors in our transit light curve analysis (described below in Section 5). We upgraded LDTk to allow the (typically nonGaussian) posterior distributions generated by the isochrones package to be fed directly into the limb-darkening analysis. ${ }^{40}$ Because LDTk often reports implausibly small uncertainties on the limb-darkening parameters, based on our experience with such analyses we increase all these uncertainties by a factor of five in our light curve analyses. Spot checks of a number of systems reveal that imposing priors on the stellar limb-darkening has a negligible impact $(<1 \sigma)$ on our final results, relative to analyses with much weaker constraints on limb darkening.

All our derived stellar parameters- $T_{\mathrm{eff}}, \log g, R_{\star}, M_{\star}$-and their uncertainties are listed in Table 7.

$\overline{{ }^{40} \text { GitHub commits 60174cc, 46d140b, and } 8927 b c 6 .}$

\section{TRANSIT LIGHT CURVE ANALYSES}

After identifying planet candidates and determining the parameters of their host stars, we subject the detrended light curves to a full maximum-likelihood and MCMC analysis. We use a custom Python wrapper of the free, open source BATMAN light curve code (Kreidberg 2015). We upgraded the BATMAN codebase to substantially increase its efficiency when analyzing long-cadence data ${ }^{41}$ The light curves are fit using the standard Nelder-Mead Simplex Algorithm ${ }^{42}$ and then run through emcee to determine parameter uncertainties.

Our general approach follows that used in our previous papers (Crossfield et al. 2015; Petigura et al. 2015; Schlieder et al. 2016; Sinukoff et al. 2016). The model parameters in our analysis are the transit time $\left(T_{0}\right)$; the candidate's orbital period and inclination $(P$ and $i)$; the scaled semimajor axis $\left(a / R_{*}\right)$; the fractional candidate size $\left(R_{p} / R_{*}\right)$; the orbital eccentricity and longitude of periastron $(e$ and $\omega)$, the fractional level of dilution $(\delta)$ from any other sources in the aperture; a single multiplicative offset for the absolute flux level; and quadratic limbdarkening coefficients $\left(u_{1}\right.$ and $\left.u_{2}\right)$. We initialize each fit with the best-fit parameters returned from TERRA. Note that both this analysis and that of TERRA assume a linear ephemeris, so systems with large transit timing variations (TTVs) could be missed or misidentified.

During the analysis, several parameters are constrained or subjected to various priors. Gaussian priors are applied to the limb-darkening parameters (as derived from the LDTk analysis), to $P$ (with a dispersion of $\sigma_{P}=0.01$ days, to ensure that the desired candidate signal is the one being analyzed), and to $e\left(\mu_{e}=10^{-4}\right.$ and $\sigma_{e}=10^{-3}$, to enforce a circular orbit). We also apply a uniform prior to $T_{0}$ (with width $0.06 P$ ), $i$ (from $50^{\circ}$ to $90^{\circ}$ ), $R_{p} / R_{*}$ (from -1 to 1 ), and $\omega$ (from 0 to $2 \pi$ ); both $P$ and $a / R_{*}$ are furthermore constrained to be positive. Allowing $R_{P} / R_{*}$ to take on negative values avoids the Malmquist bias that would otherwise result from treating it as a positivedefinite quantity. For those systems with no identified stellar companions, our HRI and/or spectroscopy constrain the dilution level; otherwise, we adopt a log-uniform prior on the interval $\left(10^{-6}, 1\right)$.

\section{FALSE POSITIVE ASSESSMENT}

During the prime Kepler mission, both the sheer number of planet candidates and their intrinsic faintness made direct confirmation by RVs impractical for most systems. Nonetheless many planets can be statistically validated by assessing the relative probabilities of planetary and false positive scenarios; a growing number of groups have presented frameworks for quantitatively assessing the likelihoods of planetary and false positive scenarios (Torres et al. 2011; Morton 2012; Díaz et al. 2014; Santerne et al. 2015). These false positive scenarios come in several classes: (1) undiluted eclipsing binaries, (2) background (and foreground) eclipsing binaries where the eclipses are diluted by a third star, and (3) eclipsing binaries in gravitationally bound triple systems.

To estimate the likelihood that each of our planet candidates is a true planetary system or a false positive configuration, we use the free and open source vespa software (Morton 2015). vespa compares the likelihood of each scenario against the planetary interpretation and accepts additional constraints from

\footnotetext{
${ }^{41}$ GitHub commit 9ae9c83.

42 As implemented in scipy.optimize.fmin.
} 
Table 7

Stellar Parameters

\begin{tabular}{|c|c|c|c|c|c|c|}
\hline EPIC & $\begin{array}{c}\mathrm{Kp} \\
(\mathrm{mag})\end{array}$ & $\begin{array}{c}R_{*} \\
\left(R_{\odot}\right)\end{array}$ & $\begin{array}{c}M_{*} \\
\left(M_{\odot}\right)\end{array}$ & $\begin{array}{l}T_{\text {eff }} \\
(\mathrm{K})\end{array}$ & $\begin{array}{l}\log g \\
(\operatorname{dex})\end{array}$ & Source \\
\hline 201155177 & 14.632 & $0.643(39)$ & $0.702(46)$ & $4613(71)$ & $4.659(50)$ & Huber et al. (2016) \\
\hline 201208431 & 14.409 & $0.435(60)$ & $0.487(72)$ & $4044(81)$ & $4.849(60)$ & Huber et al. (2016) \\
\hline 201247497 & 16.770 & $0.436(27)$ & $0.492(29)$ & $3918(46)$ & $4.846(50)$ & Huber et al. (2016) \\
\hline 201295312 & 12.126 & $1.58(15)$ & $1.150(60)$ & $5912(51)$ & $4.101(63)$ & SpecMatch \\
\hline 201345483 & 15.319 & $0.445(66)$ & $0.503(78)$ & 4103(90) & $4.824(70)$ & Huber et al. (2016) \\
\hline 201367065 & 11.574 & $0.371(50)$ & $0.414(58)$ & $3841(82)$ & $4.906(60)$ & Huber et al. (2016) \\
\hline 201384232 & 12.510 & $1.010(80)$ & $0.930(30)$ & $5767(58)$ & $4.398(74)$ & SpecMatch \\
\hline
\end{tabular}

(This table is available in machine-readable form.)

HRI and spectroscopy. Throughout this analysis, we apply Version 0.4 .7 of vespa (using the MultiNest backend) to each individual planetary candidate. Other types of false positive scenarios exist that are not explicitly treated by vespa, such as eclipsing binaries on eccentric, inclined orbits showing only transit or occultation (but not both), or extremely inconvenient arrangements of starspots. The community's experience of following up transiting planet candidates indicates that such scenarios are much less common than those considered by vespa; nonetheless quantifying the likelihood of such scenarios for each candidate would be an interesting avenue for future research.

\subsection{Calculating FPPS}

To calculate the FPP for each system, we use the following inputs: stellar photometry from APASS, 2MASS, and WISE; the stellar parameters described in Section 4; the detrended light curve (after masking out any transits from other candidates in that system); the exclusion constraints from adaptive optics imaging data in terms of contrast versus separation (where available) and from our high-resolution spectroscopy (maximum allowed contrast and velocity offset); and an upper limit on the depth of any secondary eclipse. We derive the last of these by constructing a rectangular signal with depth unity and duration equal to the best-fit transit duration, scanning the template signal across the out-of-transit light curve, and reporting the 99.7th percentile as the eclipse depth's upper limit.

We report the final FPPs of all our systems in Table 8. For the purposes of the discussion that follows, we deem any candidate signal with FPP $<0.01$ as a validated extrasolar planet and signals with FPP $>0.99$ as false positives. For all unvalidated candidates, Table 9 summarizes vespa's estimate of the relative (unnormalized) likelihood of each potential false positive scenario.

The vespa algorithm implicitly assumes that each planet candidate lacks any other companion candidates in the same system. Studies of Kepler's multiple-candidate systems show that almost all are planets (Lissauer et al. 2012). This "multiplicity boost" has subsequently been used to validate hundreds of multi-planet systems (Rowe et al. 2014). Because vespa treats only single-planet systems, we simply treat these multi-candidate systems as independent, isolated candidates in the FPP analysis. Sinukoff et al. (2016) show that K2's multiplicity boost is $\geqslant 20$ even in crowded fields, comparable to the boost factor derived for the original Kepler mission.

Even without the multiplicity boost, our approach validates the majority of our multi-candidate systems. Both EPIC 201445392 (K2-8) and EPIC 206101302 host two-planet candidates. In each system we validate one candidate and find FPP $=4 \%-7 \%$ for the other. The $K 2$ multiplicity boost factor of $\geqslant 20$ therefore results in all candidates in both systems being firmly labeled as validated planets.

A more complicated case is EPIC 205703094, which hosts three planet candidates. Our vespa analysis finds that one candidate is a false positive and that the others both have FPP $\approx 50 \%$ (see Tables 8 and 9). Our light curve analysis finds that all three candidates are well-fit by grazing transits $(b \sim 1)$, leaving $R_{P} / R_{*}$ only weakly constrained. Furthermore, our HRI reveals that the system is a close visual binary with separation 0 ". 14 (see Tables 6 and 5). Therefore we can neither validate nor rule out the three candidates in this system.

\subsection{Targets with Nearby Stellar Companions}

Planet candidates orbiting stars in physical or visual multiple systems are much more difficult to validate due to blending in the photometric aperture (see, e.g., Ciardi et al. 2015). Table 5 shows that our $K 2$ photometric apertures are quite large (up to $20^{\prime \prime}$ in extreme cases) and that HRI follow-up reveals stellar companions within these apertures for many systems. Therefore we must treat these systems with greater care.

To demonstrate the difficulty, consider two stars with flux ratio $F_{2} / F_{1}<1$ and angular separation $\rho$. Assume both lie in a photometric aperture with radius $r>\rho$, with which a transit is observed with apparent depth $\delta^{\prime}$. If the transit occurs around the primary star, then the true transit depth is $\delta_{1} \approx \delta^{\prime} /\left(1-F_{2} / F_{1}\right)$; this is at most twice the observed depth, indicating a planetary radius up to $\sqrt{2}$ larger than otherwise determined. If instead the transiting object orbits the secondary, then the true transit depth is $\delta_{2} \approx \delta^{\prime} F_{1} / F_{2}$ and the transiting object may be many times larger than expected. Table 6 lists all candidates known to host secondary stars and their relationships between $F_{2} / F_{1} \& \delta^{\prime}$ and $\rho \& r$.

Any planet candidate in a multi-star system and with $F_{2} / F_{1}<\delta^{\prime}$ cannot transit the secondary (which is too faint to be the source of the observed transit signal). We find several 
Table 8

Planet Candidate Parameters

\begin{tabular}{|c|c|c|c|c|c|c|c|c|c|c|}
\hline $\begin{array}{l}\text { Other } \\
\text { Name }\end{array}$ & EPIC & $\begin{array}{c}P \\
\text { (d) }\end{array}$ & $\begin{array}{c}T_{0} \\
\left(\mathrm{BJD}_{\mathrm{TDB}}\right. \\
-2454833)\end{array}$ & $\begin{array}{l}T_{14} \\
\text { (hr) }\end{array}$ & $\begin{array}{c}R_{P} / R_{*} \\
(\%)\end{array}$ & $\begin{array}{c}a \\
(\mathrm{au})\end{array}$ & $\begin{array}{c}R_{P} \\
\left(R_{\oplus}\right)\end{array}$ & $\begin{array}{c}S_{\mathrm{inc}} \\
\left(S_{\oplus}\right)\end{array}$ & FPP & Disposition \\
\hline \multirow[t]{2}{*}{ K2-42b } & 201155177.01 & $6.68796(93)$ & $1981.6763(52)$ & $3.04(20)$ & $3.04(28)$ & $0.0617(13)$ & $2.15(24)$ & $44.1(6.3)$ & $5.2 \mathrm{e}-07$ & Planet \\
\hline & 201176672.01 & 79.9999(98) & $2044.8046(15)$ & $11.75(17)$ & $18.0(1.1)$ & $0.299(16)$ & $10.2(2.1)$ & $1.10(46)$ & 0.094 & Candidate (see Tables 6 and 1 ) \\
\hline $\mathrm{K} 2-43 \mathrm{~b}$ & 201205469.01 & $3.47114(21)$ & $1976.8845(33)$ & $1.97(11)$ & $6.60(36)$ & $0.03784(63)$ & $4.13(31)$ & $49.0(6.9)$ & $9.7 \mathrm{e}-10$ & Planet \\
\hline \multirow[t]{2}{*}{$\mathrm{K} 2-4 \mathrm{~b}$} & 201208431.01 & $10.0044(11)$ & $1982.5170(45)$ & $2.87(17)$ & $3.49(41)$ & $0.0715(35)$ & $1.69(30)$ & $8.9(2.7)$ & 0.00028 & Planet \\
\hline & 201247497.01 & $2.75391(15)$ & $1977.9031(23)$ & $1.03(14)$ & $7.9(1.3)$ & $0.03035(59)$ & $3.78(68)$ & $43.7(6.1)$ & 0.41 & Candidate \\
\hline \multirow[t]{2}{*}{$\mathrm{K} 2-44 \mathrm{~b}$} & 201295312.01 & $5.65688(59)$ & $1978.7176(44)$ & $4.36(13)$ & $1.56(12)$ & $0.0651(11)$ & $2.72(32)$ & $646(126)$ & $6.7 \mathrm{e}-07$ & Planet \\
\hline & 201324549.01 & $2.519334(35)$ & $1976.99353(69)$ & $1.545(15)$ & $2.41(30)$ & $0.0383(13)$ & $3.91(85)$ & $2002(727)$ & 1 & False Positive (see Tables 6 and 3 ) \\
\hline $\mathrm{K} 2-5 \mathrm{c}$ & 201338508.01 & $10.9324(14)$ & $1981.6012(55)$ & $2.84(23)$ & $3.24(29)$ & $0.0775(22)$ & $1.64(20)$ & $8.3(1.6)$ & $2.8 \mathrm{e}-05$ & Planet \\
\hline $\mathrm{K} 2-5 \mathrm{~b}$ & 201338508.02 & $5.73597(68)$ & $1975.8602(53)$ & $2.35(18)$ & $2.97(22)$ & $0.0504(14)$ & $1.50(17)$ & $19.7(3.7)$ & $5.4 \mathrm{e}-06$ & Planet \\
\hline $\mathrm{K} 2-45 \mathrm{~b}$ & 201345483.01 & $1.7292684(69)$ & $1976.52604(18)$ & $1.689(14)$ & $13.76(19)$ & $0.0224(12)$ & $6.71(00)$ & $100(32)$ & $6.8 \mathrm{e}-06$ & Planet \\
\hline $\mathrm{K} 2-3 \mathrm{~b}$ & 201367065.01 & $10.05443(26)$ & $1980.4178(12)$ & $2.520(59)$ & $3.51(15)$ & $0.0679(32)$ & $1.44(20)$ & $5.8(1.7)$ & $1.9 \mathrm{e}-08$ & Planet \\
\hline $\mathrm{K} 2-3 \mathrm{c}$ & 201367065.02 & $24.6435(12)$ & $1979.2811(24)$ & $3.38(12)$ & $2.88(16)$ & $0.1235(58)$ & $1.18(17)$ & $1.77(53)$ & $2.1 \mathrm{e}-08$ & Planet \\
\hline $\mathrm{K} 2-3 \mathrm{~d}$ & 201367065.03 & $44.5609(52)$ & $1993.2285(34)$ & 4.04(19) & $2.35(15)$ & $0.1833(86)$ & $0.96(14)$ & $0.80(24)$ & $1.2 \mathrm{e}-07$ & Planet \\
\hline
\end{tabular}

Note. Table 8 is published in its entirety in the machine-readable format. A portion is shown here for guidance regarding its form and content.

(This table is available in its entirety in machine-readable form.) 
Table 9

Unvalidated Candidate False Positive Likelihoods

\begin{tabular}{|c|c|c|c|c|c|c|c|c|}
\hline$\overline{\text { Target }}$ & L_heb $^{\mathrm{a}}$ & "L_heb_P $\times 2^{\text {a }}$ & $\overline{L_{-} \mathrm{eb}^{\mathrm{b}}}$ & 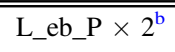 & $\overline{\text { L_beb }{ }^{\mathrm{c}}}$ & L_beb_P $\times 2^{\text {c }}$ & L__pl ${ }^{\mathrm{d}}$ & FPP \\
\hline 201176672.01 & $3.2 \mathrm{e}-36$ & 0.0097 & $7.3 e-19$ & 0.046 & 0.002 & 0.0019 & 0.035 & 0.094 \\
\hline 201247497.01 & $7.2 \mathrm{e}-16$ & $7.4 e-05$ & 2.6 & 20 & 0 & 2.9 & 12 & 0.41 \\
\hline 201445392.01 & 0.00027 & 0.028 & 0.042 & 0.83 & 0 & 0.081 & 1.5 & 0.042 \\
\hline 201465501.01 & 0.00047 & 0.19 & 0.0036 & 0.46 & 0 & 0.015 & 0.042 & 0.45 \\
\hline 201512465.01 & 0.0021 & 0.09 & 0.013 & 0.82 & 1.3 & 5.6 & 8.3 & 0.23 \\
\hline 201546283.01 & $6 e-15$ & $1 e-07$ & $8.1 \mathrm{e}-10$ & $5.4 \mathrm{e}-16$ & 0.0019 & 0.00045 & 14 & $4.4 \mathrm{e}-07$ \\
\hline 201565013.01 & $3.6 \mathrm{e}-07$ & 0.073 & 16 & 1.2 & 1.1 & 0.44 & 8 & 0.76 \\
\hline 201617985.01 & 0.0048 & 0.77 & 0.0046 & 1.5 & 0.09 & 0 & 0.025 & 0.96 \\
\hline 201626686.01 & $5.5 e-20$ & $1.1 \mathrm{e}-10$ & 0.3 & 0.012 & $4.6 e-05$ & $5.6 e-07$ & 1.6 & 0.16 \\
\hline 201629650.01 & $5.9 \mathrm{e}-69$ & $2.3 e-21$ & $1.2 \mathrm{e}-07$ & $1.1 \mathrm{e}-08$ & $5.2 \mathrm{e}-11$ & $1.2 \mathrm{e}-39$ & 0.002 & $3.6 \mathrm{e}-05$ \\
\hline 201637175.01 & 4 & 1.3 & 0.23 & 15 & 0.17 & 2.2 & 19 & 0.15 \\
\hline 201702477.01 & $1.6 \mathrm{e}-12$ & $2.9 \mathrm{e}-06$ & 0.13 & 0.0048 & 0.19 & 0.021 & 0.79 & 0.41 \\
\hline 201717274.01 & $7 e-12$ & $1.6 \mathrm{e}-05$ & $3.9 \mathrm{e}-08$ & 0.00016 & 0.25 & 0.71 & 8.9 & 0.013 \\
\hline 201828749.01 & $1.4 \mathrm{e}-14$ & $1.1 \mathrm{e}-13$ & $5.4 \mathrm{e}-09$ & $5.3 e-28$ & 0 & 0 & 0.059 & $3.3 e-08$ \\
\hline 201862715.01 & $2.2 \mathrm{e}-23$ & $4 e-09$ & $6.8 \mathrm{e}-18$ & $4.5 \mathrm{e}-13$ & 0.014 & $2.3 e-07$ & 5.8 & $2.4 \mathrm{e}-07$ \\
\hline 201920032.01 & $7.9 \mathrm{e}-34$ & $2.4 \mathrm{e}-10$ & 0.078 & 0.056 & 0.0014 & 0 & 0.99 & 0.054 \\
\hline 202071289.01 & 0.076 & 0.96 & 4.5 & 3.5 & 0.00015 & $1.9 \mathrm{e}-08$ & 11 & 0.97 \\
\hline 202071401.01 & 0.0062 & 0.16 & 0.0086 & 0.27 & 0 & $7.1 \mathrm{e}-05$ & 2.5 & 0.002 \\
\hline 202126852.01 & 0.059 & 0.0024 & 4.2 & 0.036 & 0 & 0.015 & 2.8 & 0.65 \\
\hline 202675839.01 & 0.045 & 0.19 & 0.18 & 0.24 & $1 e-14$ & 0 & 0.46 & 0.19 \\
\hline 205029914.01 & $2 e-34$ & $5.4 \mathrm{e}-10$ & $1 e-34$ & $4.7 \mathrm{e}-16$ & 0 & $3.3 e-12$ & 0.0054 & $1.4 \mathrm{e}-10$ \\
\hline 205148699.01 & $5.4 \mathrm{e}-58$ & $4.7 e-34$ & $6.5 e-31$ & $1.2 \mathrm{e}-48$ & 0 & 0 & 0.81 & 0 \\
\hline 205570849.01 & 0.041 & 0.38 & 0.78 & 0.48 & 0 & $4.7 \mathrm{e}-25$ & 1.2 & 0.32 \\
\hline 205686202.01 & $1.8 \mathrm{e}-36$ & $3.2 \mathrm{e}-16$ & $2.7 e-40$ & $7 e-11$ & $2 \mathrm{e}-08$ & 0 & 2.5 & $1.3 \mathrm{e}-11$ \\
\hline 205703094.02 & 0.0027 & 0.049 & 0.0011 & 0.034 & 0.0073 & 0.0097 & 0.13 & 0.66 \\
\hline 205999468.01 & 0.056 & 0.85 & 0.96 & 1.2 & 0.43 & 1.1 & 2.8 & 0.1 \\
\hline 206011496.01 & $4.1 \mathrm{e}-26$ & $1.7 \mathrm{e}-06$ & $4.4 \mathrm{e}-15$ & $1.4 \mathrm{e}-05$ & 0 & 0 & 12 & $1.6 \mathrm{e}-08$ \\
\hline 206024342.01 & $3 e-07$ & $6.6 e-06$ & 0.021 & 0.027 & 0 & 0 & 0.84 & 0.025 \\
\hline 206028176.01 & 0.0024 & 0.072 & 0.015 & 0.1 & 0 & 0 & 0.0065 & 0.64 \\
\hline 206036749.01 & 1.3 & 1.3 & 1.6 & 0.59 & 0 & 0 & 2.1 & 0.097 \\
\hline 206061524.01 & $4.7 \mathrm{e}-22$ & $1 e-08$ & $1.1 \mathrm{e}-16$ & $3.7 \mathrm{e}-18$ & 0.0023 & $5 e-08$ & 36 & $4.6 \mathrm{e}-08$ \\
\hline 206101302.01 & 0.00036 & 0.0023 & 0.051 & 0.085 & 0.072 & 0 & 0.61 & 0.069 \\
\hline 206114294.01 & 0.0083 & 0.16 & 0.46 & 2 & 0 & 0 & 19 & 0.028 \\
\hline 206154641.01 & 0.00065 & 0.016 & 2.1 & 0.059 & 0 & 0 & 15 & 0.074 \\
\hline 206192335.01 & $1.4 \mathrm{e}-08$ & 0.0042 & $2.1 \mathrm{e}-05$ & 0.022 & 0 & 0 & 11 & $6 e-05$ \\
\hline 206247743.01 & $8.7 \mathrm{e}-78$ & $2.6 e-22$ & $8.5 e-26$ & $3.2 \mathrm{e}-06$ & 0 & 0 & $2.3 e-06$ & 0.025 \\
\hline 206403979.01 & $1.2 \mathrm{e}-08$ & $8.5 e-07$ & 0.13 & 0.06 & 0.015 & 0.012 & 0.26 & 0.72 \\
\hline 206543223.01 & 0.045 & 0.25 & 1.2 & 0.0046 & 0.0016 & 0.021 & 2.2 & 0.67 \\
\hline 207739861.01 & $3.5 e-203$ & $1.4 \mathrm{e}-80$ & $1.4 \mathrm{e}-66$ & $2.6 e-21$ & 0 & 0 & $2.4 \mathrm{e}-05$ & $1.1 \mathrm{e}-16$ \\
\hline 208833261.01 & 0 & $8.9 \mathrm{e}-196$ & $1.1 \mathrm{e}-05$ & 0.00091 & 0.23 & 0.25 & 2.5 & 0.011 \\
\hline 209036259.01 & 0 & $4 e-40$ & $3.9 \mathrm{e}-19$ & 0.075 & 0 & 0 & 0.00059 & 0.96 \\
\hline 210389383.01 & $4.3 e-77$ & $3.8 \mathrm{e}-64$ & $5.2 \mathrm{e}-07$ & $6.2 \mathrm{e}-39$ & $6.9 e-05$ & $3.4 \mathrm{e}-09$ & 2.4 & $4.1 \mathrm{e}-05$ \\
\hline 210609658.01 & $2.5 \mathrm{e}-146$ & $2.3 e-20$ & $3.3 e-13$ & 0.0098 & $3.7 e-18$ & $6.6 e-12$ & 0.0077 & 0.015 \\
\hline 210625740.01 & 2.3 & 0.86 & 0.18 & 5 & 0.036 & 1.5 & 31 & 0.13 \\
\hline 210659688.01 & 0.017 & 0.012 & 0.016 & 0.073 & 0.016 & 0.023 & 0.17 & 0.25 \\
\hline 210666756.01 & 5.3 & 0 & 0.42 & 2.8 & 0.055 & 0.37 & 2.4 & 0.013 \\
\hline 210754505.01 & $1 e-07$ & 0.015 & 15 & 0.8 & 0 & 1.6 & 1.5 & 0.24 \\
\hline 210903662.01 & 4.1 & 5.2 & 12 & 7.9 & 0.61 & 0 & 7 & 0.59 \\
\hline 210958990.01 & 3.8 & 0.0015 & 0.01 & $9.1 \mathrm{e}-16$ & 0.41 & $1.1 \mathrm{e}-09$ & 0.032 & 0.98 \\
\hline 211147528.01 & 0.018 & 0.00047 & 0.45 & 0.003 & 0.47 & 0.053 & 1.8 & 0.26 \\
\hline 211916756.01 & $1.3 e-33$ & $2.7 \mathrm{e}-09$ & $1 e-28$ & $2.3 e-09$ & 0.9 & 0.43 & 0.003 & 0.88 \\
\hline
\end{tabular}

Notes.

${ }^{a}$ Likelihood that the system is a hierarchical eclipsing binary, with orbital period either as measured or twice that measured.

${ }^{\mathrm{b}}$ Likelihood that the system is an eclipsing binary, with orbital period either as measured or twice that measured.

${ }^{\mathrm{c}}$ Likelihood that the system is a blended eclipsing binary, with orbital period either as measured or twice that measured.

${ }^{d}$ Likelihood that the system is a transiting planet.

(This table is available in machine-readable form.)

such systems, though only two (EPIC 202126852 and 211147528) have FPP $<0.95$. Nonetheless, for all these systems we account for the dilution of the secondary star(s) as described below.
For candidates with $\delta^{\prime}<F_{2} / F_{1}$ and $\rho<r$, the transit could occur around either star. We compare our nominal time-series photometry to that computed with $r=1$ pixel for all such candidates. For targets with more widely separated nearby 
Table 10

High-resolution Imaging

\begin{tabular}{lccl}
\hline \hline EPIC & Filter & $t_{\text {int }}(\mathrm{s})$ & Instrument \\
\hline 201155177 & $\mathrm{~K}$ & 330 & NIRI \\
201176672 & $\mathrm{~K}$ & 270 & NIRC2 \\
201205469 & $\mathrm{~K}$ & 810 & NIRC2 \\
201208431 & $\mathrm{~K}$ & 171 & NIRC2 \\
201247497 & $\mathrm{~K}$ & 540 & NIRC2 \\
201295312 & $\mathrm{~K}$ & 212.4 & PHARO \\
201295312 & $\mathrm{~K}$ & 225 & NIRC2 \\
201324549 & $\mathrm{~K}$ & 276.1 & PHARO \\
201324549 & $\mathrm{~K}$ & 300 & NIRI \\
201338508 & $\mathrm{~K}$ & 1080 & NIRC2 \\
\hline
\end{tabular}

(This table is available in its entirety in machine-readable form.)

stars, if the one-pixel-photometry reveals a shallower transit, then the transit probably occurs around the secondary star. However, if $\rho<1$ pix then we cannot reliably identify the source of the transits. We find 28 candidates of these types that we cannot validate at present, and note the disposition of all such systems in Table 6.

For all remaining systems, the detected transits must occur around the primary star but will be diluted by light from the secondary. We estimate the total brightness of these systems' secondary star(s) as follows. For stars detected by optical imaging (Robo-AO and DSSI), we use the measured contrast ratio with an uncertainty of $0.05 \mathrm{mag}$. For stars detected by infrared imaging, we use the relations of Howell et al. (2012) to translate the observed infrared color into the Kepler bandpass. Since these relations are approximate and depend strongly on $\mathrm{SpT}$, we conservatively apply an uncertainty of $0.5 \mathrm{mag}$ to these values. Section 6.2 describes how we use these data to constrain the dilution parameter's posterior distribution, thereby reducing the systematic biases induced by unrecognized sources of dilution (e.g., Ciardi et al. 2015).

\section{RESULTS AND DISCUSSION}

We find 104 validated planets (i.e., FPP $<0.01$ ) in our set of 197 planet candidates. Significantly, we show that $K 2$ 's surveys increase by $30 \%$ the number of small planets orbiting moderately bright stars compared to previously known planets. In Section 7.1 we present a general overview of our survey results. Then, in Section 7.2 we discuss individual systems, both new targets and previously identified planets and candidates.

\subsection{Overview of Results}

Our validated planetary systems span a range of properties, with median values of $R_{P}=2.3 R_{\oplus}, \quad P=8.6$ days, $T_{\text {eff }}=5300 \mathrm{~K}$, and $K p=12.7 \mathrm{mag}$. Figure 7 shows the distribution of planet radius, orbital period, and final disposition for our entire candidate sample. The candidates range from 0.7 to 44 days, and from $<1 R_{\oplus}$ to larger than any known planets.

Figure 8 shows that the majority of candidates have $R_{P}<3 R_{\oplus}$, and these smallest candidates exhibit the highest validation rates. In contrast, we validate less than half of candidates with $R_{P}>3 R_{\oplus}$ and less than half of candidates with $P<2$ days (Figure 1 ). We find a substantially higher validation rate for target stars cooler than $\sim 5500 \mathrm{~K}$ versus hotter stars (65\% versus 37\%; see Figure 9). Figure 10 shows that we validate no systems with $K p>16 \mathrm{mag}$, but otherwise reveals no obvious trends with stellar brightness.

Our analyses leave 63 planet candidates with no obvious disposition (i.e., $0.01<$ FPP $<0.99$ ). These candidates are typically large $\left(R_{P}>3 R_{\oplus}\right)$, and their FPPs are listed in Table 8 . Furthermore, in Table 9 we list the individual likelihoods of each false positive scenario considered by vespa.

We calculate the FPR of our entire planet candidate sample by taking our 197 candidates, excluding the 28 candidates with nearby stars discovered by HRI that we cannot validate (see Section 6.2), and integrating over the probability that each candidate is a planet. In this way we estimate that our entire sample contains roughly 145 total planets (though we validate just 104). This ratio corresponds to a false positive rate of 15\%-30\%, with higher FPPs for candidates showing larger sizes and/or shorter orbital periods (see Figures 1 and 8).

We also split our sample into several bins in radius and period to estimate the FPR for each subset, listed in Table 2. Our FPR is dominated by larger candidates, just as Figure 8 suggests. Sub-Jovian candidates (with $R_{P} \leqslant 8 R_{\oplus}$ ) have a cumulative FPR of $\sim 10 \%$, whereas over half of the larger candidates are likely false positives. The FPR for larger candidates is consistent with that measured for the original Kepler candidate sample (Santerne et al. 2016b). Candidates with $P<3$ days have a FPR roughly twice as high as that for longer-period systems.

Since we have excluded the 28 candidates described above, these FPRs are only approximate and we defer a more detailed analysis of our survey completeness and accuracy to a future publication. Nonetheless, further follow-up observations for systems lacking high-resolution spectroscopy, HRI, and/or RV measurements may expect to identify, validate, and confirm a considerable number of additional planetary systems.

Figure 11 shows planet radius versus the irradiation levels incident upon each of our validated planets relative to that received by the Earth $\left(S_{\oplus}\right)$, color-coded by $T_{\text {eff }}$. These planets receive a wide range of irradiation, from roughly that of Earth to over $10^{4} \times$ greater. As expected, our coolest validated planets orbit cooler stars ( $\mathrm{K}$ and $\mathrm{M}$ dwarfs). However, we caution that the stellar parameters for these systems come from broadband colors and/or Huber et al. (2016), so uncertainties are large and biases may remain. Follow-up spectroscopy is underway to more tightly constrain the stellar and planetary properties of these systems (C. Dressing et al. 2016, in preparation; A. Martinez et al. 2016, in preparation).

Finally, Figure 12 shows that $K 2$ planet survey efforts have substantially increased the number of smaller planets known to orbit moderately bright stars. Although our sensitivity appears to drop off below $\sim 1.3 R_{\oplus}$ (as shown in Figure 8 ) and we find no planets around stars brighter than $J<8.9 \mathrm{mag}$, we validate a substantial number of intermediate-size planets around moderately bright stars. In particular, the right panel of Figure 12 shows that the first five fields of $K 2$ have already increased the number of small planets orbiting fairly bright stars by roughly $30 \%$ compared to those tabulated at the NASA Exoplanet Archive. Considering the sizes of these planets and the brightness of their host stars, many of these systems are amenable to follow-up characterization via Doppler spectroscopy and/or JWST transit observations. 

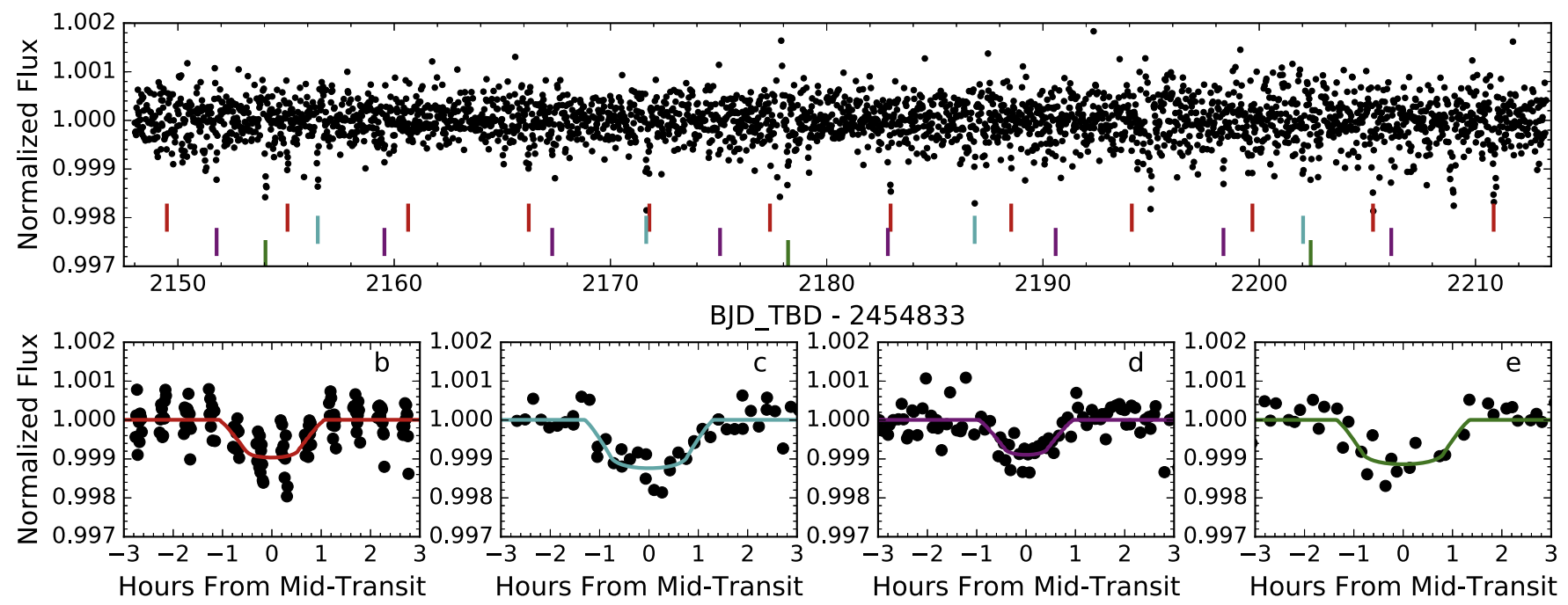

Figure 6. Photometry of K2-72 (EPIC 206209135), which hosts four transiting planets. Top: full time-series, with colored tick marks indicating each individual transit time. Bottom: phase-folded photometry with the color-coded, best-fit transit model overplotted for each planet. Our analysis indicates a stellar radius of $0.40_{-0.07}^{+0.12} R_{\odot}$, planetary radii of $1.2-1.5 R_{\oplus}$, and (from left to right) orbital periods of $5.58,7.76,15.19$, and 24.16 days.

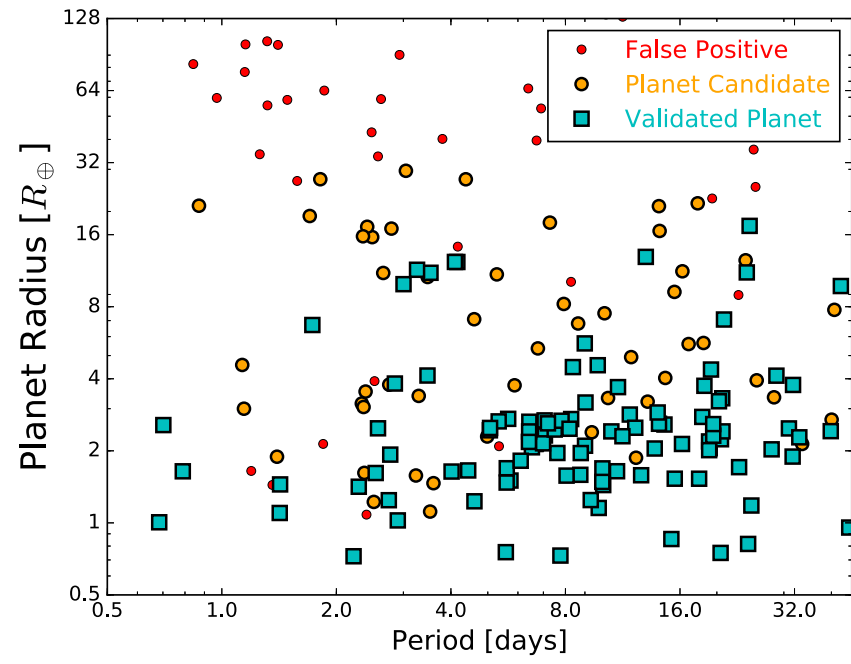

Figure 7. Orbital periods and radii of our 104 validated planets, 30 false positive systems, and 63 remaining planet candidates. Uncertainties on planet radius (listed in Table 8 ) are typically $\sim 13 \%$.

\subsection{Notes on Individual Systems}

Of the 104 planets validated by our analysis, 64 are newly validated. These include several new multi-planet systems, systems as bright as $V=10.8 \mathrm{mag}$, and several small, roughly Earth-sized planets receiving roughly Earth-like levels of irradiation. Below we describe some of the most interesting new systems in Section 7.2.1, our analysis of previously confirmed or validated planets in Section 7.2.2, and our results for known but unvalidated candidates in Section 7.2.3.

\subsubsection{New Validated Planets}

K2-72 (EPIC 206209135) is a dwarf star hosting a planet candidate on a 5.57 day orbit (Vanderburg et al. 2016); we find three additional candidates and validate all four planets in this system. We see the transits in both our photometry (shown in Figure 6) and that of Vanderburg \& Johnson (2014), and our light curve fits give consistent values of $\rho_{* \text {, circ }}$ for all planetsboth points give us confidence that these are true planetary

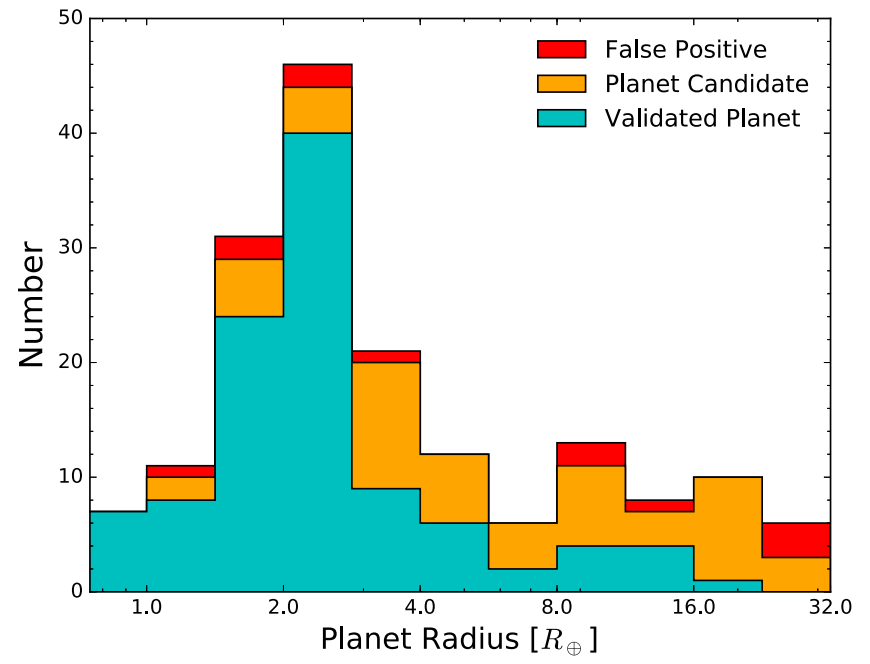

Figure 8. Distribution of planet candidate radii for our validated planets, false positive systems, and remaining planet candidates. We validate most of the candidates smaller than $3 R_{\oplus}$, consistent with the low false positive rate we find for small planets.

systems. Huber et al. (2016) reports a stellar radius of $0.23 R_{\odot}$ but notes that this is likely an underestimate. The weighted mean of our four stellar density measurements is $9.0 \pm 3.6 \mathrm{~g} \mathrm{~cm}^{-3}$; using the mass-radius relation of Maldonado et al. (2015) implies a stellar radius of $0.40_{-0.07}^{+0.12} R_{\odot}$ and planetary radii of $1.2-1.5 R_{\oplus}$ for all planets. Analysis of the stellar spectrum is also consistent with this size (C. Dressing et al. 2016, in preparation; A. Martinez et al. 2016, in preparation). These four small planets have orbital periods of 5.58, 7.76, 15.19, and 24.16 days. The irradiation levels for several planets are also quite consistent with Earth's insolation. Several of these planet pairs orbit near mean-motion resonances: planets $c$ and d orbit near the first-order 2:1 MMR, and $\mathrm{b}$ and $\mathrm{c}$ orbit near the second-order 7:5 MMR. Although the star K2-72 is relatively faint $-K p=14.4 \mathrm{mag}, K=11.0 \mathrm{mag}$ - follow-up Doppler or transit spectroscopy observations to measure the planets' masses or atmospheric compositions will be challenging; the system's near-integer period ratios suggest 


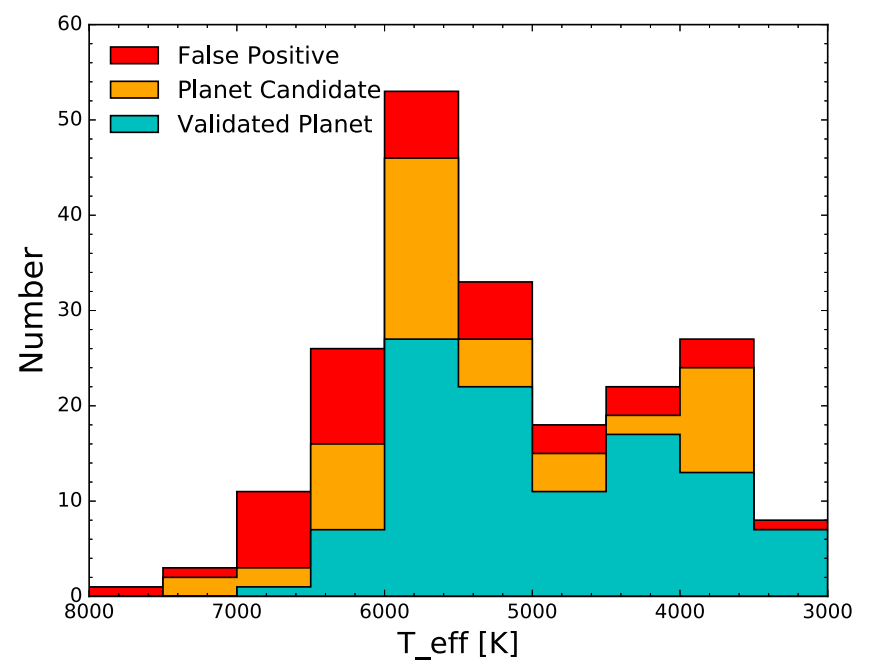

Figure 9. Distribution of stellar effective temperatures for systems with validated planets, false positive, and remaining planet candidates. There is a hint of a higher validation rate around stars cooler than $\sim 5500 \mathrm{~K}$.

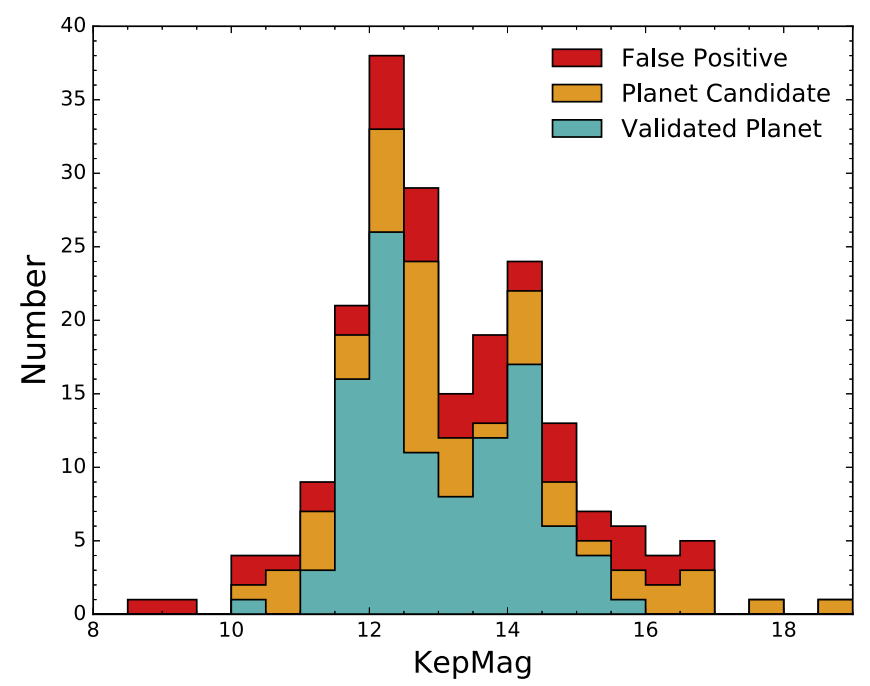

Figure 10. Distribution of $K p$ for systems with validated planets, false positives, and remaining planet candidates. Our brightest validated system, K265 (EPIC 206144956), contains a $1.6 R_{\oplus}$ planet orbiting a $V=10.8$ mag star.

that measurements of TTVs may help reveal the masses and bulk densities of all these planets.

We also identify and validate four new two-planet systems in Campaign 4: K2-80, K2-83, K2-84, and K2-90 (EPIC 210403955, 210508766, 210577548, and 210968143, respectively). Our light curve analyses of the planets in each system yield values of $\rho_{* \text {,circ }}$ that are consistent at $<1 \sigma$, consistent with the hypothesis that both planets in each pair orbit the same star. Future transit follow-up of these systems will be challenging but feasible, with the most easily observed transits having depths of $\sim 1 \mathrm{mmag}$. None of the systems appear to have planets near low-order mean-motion resonance, but additional (non-transiting) planets in these systems could lie near resonance and induce detectable TTVs.

Our brightest validated system, K2-65 (EPIC 206144956), contains a $1.6 R_{\oplus}$ planet orbiting a star with $V=10.8 \mathrm{mag}$, $J=9.0$ mag located in Campaign 3. Despite its 13-day orbital period and low predicted RV semi-amplitude (likely $\lesssim 1 \mathrm{~m} \mathrm{~s}^{-1}$ ), the bright star, relatively small planet size,

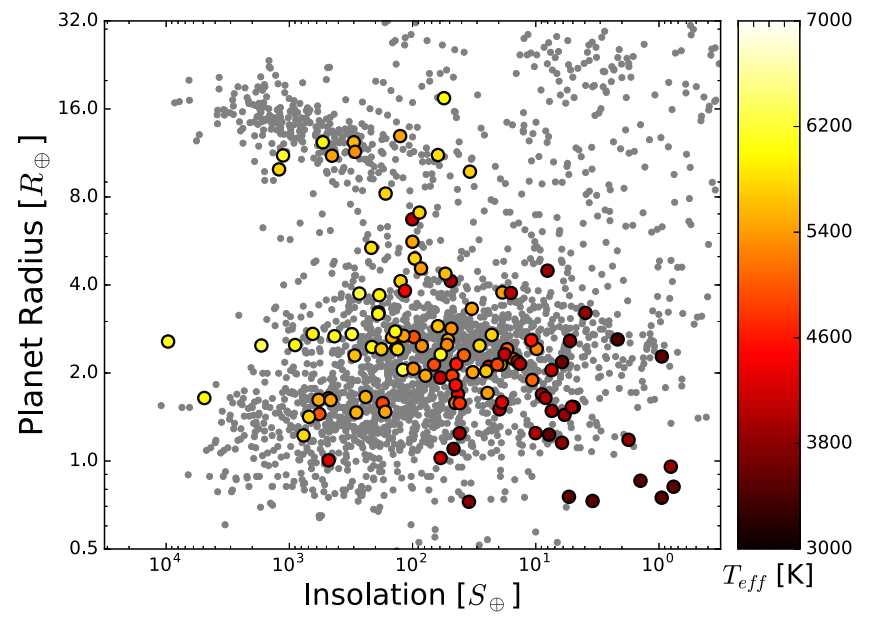

Figure 11. Planetary radii, incident insolation, and stellar effective temperature for our 104 validated planets (colored points) and all planets at the NASA Exoplanet Archive (gray points). As expected, most of our smaller, cooler planets are found around cooler, later-type stars $\left(T_{\text {eff }} \lesssim 4000 \mathrm{~K}\right)$. Uncertainties, omitted for clarity, are listed in Table 8. Statistical uncertainties on planet radius and insolation (listed in Table 8 ) are typically $\sim 13 \%$ and $\sim 26 \%$, respectively, but the coolest host stars are likely larger, hotter, and more luminous than they appear (Huber et al. 2016).

and low planet insolation (just $45 \times$ that of Earth's) may make this system an attractive target for future RV efforts.

Also of interest for RV follow-up is K2-89 (EPIC 210838726), which hosts a highly irradiated, roughly Earthsized planet on a one-day orbit around an $\mathrm{M}$ dwarf. The planet should have a RV semi-amplitude of roughly $1 \mathrm{~m} \mathrm{~s}^{-1}$, and although the star is not especially bright $(K p=13.3 \mathrm{mag}$, $K=10.1 \mathrm{mag}$ ) detection of the planet's RV signal may lie within reach of existing and planned high-precision Doppler spectrographs.

\subsubsection{Previously Confirmed Planets}

K2-3bcd and K2-26b (EPIC 201367065 and 202083828, respectively) were previously validated as sub-Neptune-sized planets orbiting M dwarfs (Crossfield et al. 2015; Schlieder et al. 2016), and K2-3b was confirmed by Doppler spectroscopy (Almenara et al. 2015). Transits of all four planets were also recently observed by Spitzer (Beichman et al. 2016). We independently validate all these planets. Note, however, that the stellar parameters we estimate here for these systems systematically underestimate the more accurate, spectroscopically derived parameters presented in those papers.

K2-10b and K2-27b (EPIC 201577035b and 201546283b, respectively) were previously validated as planets (Montet et al. 2015; Van Eylen et al. 2016a). We find FPP $<0.01$ for both, thus independently validating these two planetary systems. A new stellar companion with $\rho=3^{\prime \prime}$ and $\Delta i=5.8$ mag slightly dilutes the latter's transit but does not significantly affect its reported parameters.

We report a new stellar companion with $\rho=3$ !! 2 and $\Delta$ $K=5.8 \mathrm{mag}$ near K2-13b (EPIC 201629650; Montet et al. 2015). This new, faint star is bright enough that it could be the source of the observed transits; we therefore suggest that this previously validated system should be deemed a planet candidate.

WASP-47 (EPIC 206103150) hosts a hot Jupiter (planet b; Hellier et al. 2012), a giant planet on a 1.5 year orbit (c; Neveu- 

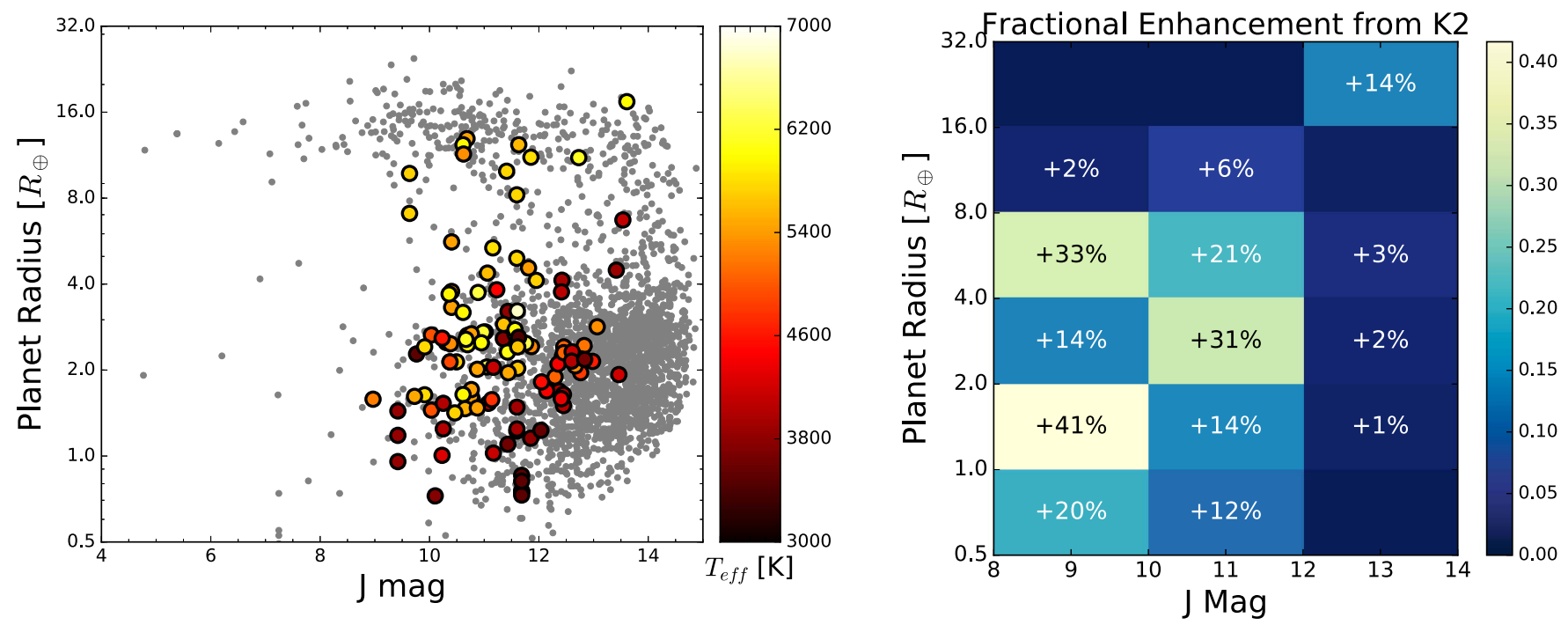

Figure 12. Left: planetary radius, stellar magnitude, and $T_{\text {eff }}$ for all validated planets (colored points) and all planets at the NASA Exoplanet Archive (gray points). Right: fractional enhancement by $K 2$ to the population of known planets. In its first five fields, $K 2$ has already substantially boosted the numbers of small, bright planets.

VanMalle et al. 2016), and two additional, short-period transiting planets (d and e; Becker et al. 2015). Our analysis of the three transiting planets yields FPP $<0.01$ for each, so we independently validate this planetary system.

HAT-P-56b (EPIC 202126852b) is a hot Jupiter confirmed by measuring the planet's mass with Doppler spectroscopy (Huang et al. 2015). Our analysis indicates that the planetary hypothesis is the most probable explanation for the signal detected, with the next-most-likely scenario being an eclipsing binary $(\mathrm{FPP}=65 \%$; see Table 9$)$. However, the RV measurements of Huang et al. (2015) rule out the eclipsing binary scenario favored by vespa and thus confirm the planetary nature of this system.

K2-19b and c (EPIC 201505350bc) were identified as a pair of planets with an orbital period commensurability near $3: 2$ (7.9 and 11.9 days; Armstrong et al. 2015; Narita et al. 2015; Barros et al. 2015). A third candidate with a period of 2.5 days was subsequently identified and validated (Sinukoff et al. 2016), which is not near any low-integer period ratios with the previously identified planets. Our analysis independently validates all three of these planets.

K2-21b and c (EPIC-206011691bc) are two planets with radii of $1.5-2 R_{\oplus}$, orbiting near a $5: 3$ orbital period commensurability (Petigura et al. 2015), and K2-24 b and c (EPIC 203771098 bc) are two low-density sub-Saturns orbiting near a 2:1 orbital period commensurability and with masses measured by Doppler spectroscopy (Petigura et al. 2016). Our analysis yields FPP $<0.01$ for all four of these planets, thereby confirming their planetary status.

K2-22b (EPIC 201637175b) is a short-period rocky planet caught in the act of disintegrating in a $9 \mathrm{hr}$ period around its host star (Sanchis-Ojeda et al. 2015). Our analysis successfully identifies this as a planet candidate and vespa indicates that the planetary hypothesis is the most probable explanation for the signal detected (FPP $=15 \%$; see Table 9). However, because vespa cannot account for this system's highly variable transit depths (from $1 \%$ to as shallow as $<10^{-3}$ ) the measured FPP is not reliable. We do not claim to de-validate $\mathrm{K} 2-22 \mathrm{~b}$.
K2-25b (EPIC 210490365) is a Neptune-sized planet transiting an M4.5 dwarf in the Hyades (Mann et al. 2016; David et al. 2016b). In our transit search, TERRA locked on to this star's 1.8 days rotation period, so we did not identify the planet candidate.

K2-31b (EPIC 204129699b) is a hot Jupiter validated by RV spectroscopy (Grziwa et al. 2015; Dai et al. 2016). Because of the grazing transit the planet radius is only poorly determined. The best-fit planet radius listed in Table 8 is implausibly large given the measured mass; this large radius likely led the vespa analysis to incorrectly assign this confirmed planet an FPP of $84 \%$.

K2-28b (EPIC 206318379b) was validated by Hirano et al. (2016) as a sub-Neptune-sized planet transiting an M dwarf. We did not identify the system in our transit search; a subsequent investigation shows that our photometry and transit search code did not properly execute for this system, and was never restarted.

K2-29b and K2-30b (EPIC 211089792 and 210957318) are hot Jupiters whose masses were recently measured via Doppler spectroscopy (Johnson et al. 2016; Lillo-Box et al. 2016; Santerne et al. 2016a). We find FPP $<0.01$ for both, and thus independently validate these systems.

The Sun-like star BD+20 594 (EPIC 210848071) is reported to host a planet with a radius of $2.3 R_{\oplus}$ on a 42-day orbit (Espinoza et al. 2016). Since $K 2$ observed only two transits of this planet, our transit search did not identify this system (see Section 2.3).

The first large sets of planet candidates and validated planets from $K 2$ were produced by Foreman-Mackey et al. (2015) and Montet et al. (2015). The former identified 36 planet candidates, of which the latter validated 21 . We successfully independently validate all but two of these planets, and find that for both outliers the disagreements are marginal. For K2-8b (EPIC 201445392b) we measure FPP $=4.2 \%$, but as discussed in Section 6 the multiplicity boost suppresses this candidate's FPP and results in a validated planet. However, we measure $\mathrm{FPP}=45 \%$ for K2-9b (EPIC 201465501), almost 10 times greater than originally reported. We attribute this difference to the stellar parameters reported for this star from our 
homogeneous isochrones stellar analysis: it reports K2-9 to be an early $\mathrm{M}$ dwarf, but a more reliable spectroscopic analysis reveals the star to be a smaller and cooler mid-M dwarf (Huber et al. 2016; Schlieder et al. 2016). The planet K2-9b is successfully validated when we use the spectroscopic parameters in our FPP analysis (Schlieder et al. 2016). The discrepancy highlights the importance of accurate and spectroscopically derived stellar parameters (especially for $\mathrm{M}$ dwarfs) when assessing planetary candidates.

Recently, our team validated several new multi-planet systems found by K2: K2-35, K2-36, K2-37, and K2-38 (EPIC 201549860, 201713348, 203826436, and 204221263; Sinukoff et al. 2016). Our analysis here uses much of the same machinery as in that work, so it should be of little surprise that we again validate all the planets in these systems.

Most recently, the giant planet K2-39b was confirmed by RV spectroscopy (Van Eylen et al. 2016b). Our analysis finds FPP $=0.025 \%$, independently demonstrating (with high likelihood) that the candidate is a planet.

\subsubsection{Previously Identified Candidates}

Several planet candidates showing just a single transit each were discovered in K2 Campaigns 1-3 (Osborn et al. 2016). Since our transit search focuses on shorter-period planets (see Section 2.3), we did not identify these systems.

K2-44 (EPIC 201295312) was identified as hosting a planet candidate by Montet et al. (2015) and Doppler spectroscopy constrains its mass to be $<12 M_{\oplus}(95 \%$ confidence; Van Eylen et al. 2016a). Our analysis of this system yields FPP $<0.01$ and thus validates this previously identified candidate.

Of the nine planet candidates identified by Montet et al. (2015), we validate five as planets: K2-44, K2-46, K2-8, K227, K2-35 (EPIC 201295312, 201403446, 201445392, 201546283, and 201549860, respectively). For three candidates (EPIC 201702477, 201617985, and 201565013), we find $0.01<$ FPP $<0.99$. For EPIC 201828749 we find FPP $<0.01$, but a nearby star seen via high-resolution imaging prevents us from validating this candidate.

The largest single sample of $K 2$ planet candidates released to date is the 234 candidates identified by Vanderburg et al. (2016) in Campaigns 0-3. Our analysis independently identifies 127 of their candidates. Of these 127, we validate 72 as planets and identify 19 as false positives. Our analysis validates several multi-planet candidate systems announced in that work: K2-23, K2-58, K2-59, K2-62, K2-63, and K2-75 (EPIC 206103150, 206026904, 206027655, 206096602, 206101302, and 206348688, respectively).

Furthermore, our analysis identifies 69 new candidates not published in the sample of Vanderburg et al. (2016); these are mostly in Campaign 4; some are in earlier Campaigns. The two samples largely overlap, but each also contains many candidates identified by only a single team. The differences between the two samples (along with our non-detection of EPIC 206318379, noted above) suggest that multiple independent analyses are essential if many planet candidates are not to be missed.

When comparing our sample with that of Vanderburg et al. (2016), we find that the largest single systematic difference between them is that they report roughly $25 \%$ more candidates with $P<5$ days. Our vetting checks suggest that most of these excess short-period planets are eclipsing binaries. In particular, our early-stage vetting procedures (described in Section 2.3) indicate that EPIC 201182911, 201270176, 201407812, 201488365, 201569483, 201649426, 202072965, 202086968, 202093020, 202843107, 203942067, 204649811, 205463986, and 206532093 are all likely false positives. Furthermore, highresolution imaging of a random selection of four of their candidate systems revealed all four to have nearby multiple stars (EPIC 203099398, 203867512, 204057095, 204750116). While these newly detected stars do not prove that the systems are false positives, they will nonetheless complicate any effort to validate these candidates.

Aside from the apparent excess of short-period false positives in the Vanderburg et al. (2016) sample, we find no statistical differences between the properties of theirs and our candidate samples. Measurements of both pipelines' detection efficiencies could determine why each team has missed so many of the candidates detected by the other group. The implication for future surveys is that multiple independent pipelines may substantially increase the total survey completeness of independent, relatively low-budget survey programs.

Adams et al. (2016) report nine new candidates in Campaigns $0-5$ with $P<1$ days. Of their five new candidates in Campaigns 0-4, we identify and validate one: K2-85b (EPIC 210707130b), which hosts a small planet on a $16 \mathrm{hr}$ period. Because our transit search did not extend to ultra-short orbital periods, we did not identify EPIC 202094740, 203533312, 210605073, or 210961508.

Schmitt et al. (2016) identify several dozen systems as likely eclipsing binaries (see their Table 1). Of these we identify four: EPIC 201324549 is a false positive, while EPIC 201626686, 204129699, and 206135267 remain candidate planets. Of their planet candidates we find three to have low FPPs EPIC 201920032, 206061524, and 206247743) and we validate five as planets (K2-55, K2-60, K2-67, K2-73, and K2-76, respectively, EPIC 205924614, 206038483, 206155547, 206245553, and 206432863). We did not identify their candidate EPIC 201516974 because of its 36.7-day orbital period.

\section{CONCLUSION AND FINAL THOUGHTS}

We have presented 104 validated planets discovered using $K 2$ photometry and supporting ground-based observations. Of these, 64 are planets validated here for the first time. Our analysis shows that $K 2$ has increased by $30 \%$ the number of small (1-4 $\left.R_{\oplus}\right)$ planets orbiting bright stars $(J=8-12 \mathrm{mag})$, as depicted in Figure 12. We report several new multi-planet systems, including the four-planet system K2-72 (EPIC 206209135); for all these systems we verify that the derived stellar parameters are consistent for each planet in each system. Our analysis finds 63 remaining planet candidates, which likely include a substantial number of planets waiting to be validated. In this work, we specifically utilize all our available follow-up data to assess the candidate systems. We claim to validate candidates only when no other plausible explanations are available; for example, many systems remain candidates because of nearby stars detected by our high-resolution imaging.

The size of our validated-planet sample demonstrates yet again the power of high-precision time-series photometry to discover large numbers of new planets, even when obtained from the wobbly platform of $K 2$. Since $K 2$ represents a natural transition from the narrow-field, long-baseline Kepler mission to the nearly all-sky, mostly short-baseline TESS survey, the 
results of our $K 2$ efforts bode well for the productivity of the upcoming TESS mission. The substantial numbers of intermediate-sized planets orbiting moderately bright stars discovered by our (and other) K2 surveys (Figure 12) will be of considerable interest for future follow-up characterization via RV spectroscopy and JWST transit observations (e.g., Greene et al. 2016).

We searched the entire sample of $K 2$ targets without regard for the different criteria used to propose all these stars as targets for the $K 2$ mission. Thus, although the planet population we present is broadly consistent with the early candidate population discovered by Kepler (e.g., Borucki et al. 2011), our results should not be used to draw conclusions about the intrinsic frequency with which various types of planets occur around different stars. To do so, we are already investigating a full end-to-end measurement of our survey completeness as a function of planet and stellar properties. By doing so, we also hope to compare the quality of the various input catalogs and selection metrics used to pick $K 2$ targets.

Both $K 2$ and TESS offer the potential for exciting new demographic studies of planets and their host stars. K2 observes a qualitatively different stellar population than Kepler, namely a much larger fraction of late-type stars (Huber et al. 2016). Stellar parameters for these late-type systems derived from photometry alone are relatively uncertain, and follow-up spectroscopy is underway to characterize these stars (Dressing et al., in prep; Martinez et al., in prep). In addition to the difference in median SpT, $K 2$ also surveys a much broader range of Galactic environments than was observed in the main Kepler mission. These two factors suggest that, once K2's detection efficiency is improved and quantified, the mission's data could address new questions about the intrinsic frequency of planets around these different stellar populations.

At present, when comparing our planets and candidates with those identified by Vanderburg et al. (2016) we find only a partial overlap between the two samples. This result could imply significant, qualitative differences in vetting effectiveness and survey completeness, and suggests that the analysis of transit survey data by multiple teams is an essential component of any strategy to maximize the number of discoveries. As noted in Section 6, we estimate that our sample has an overall FPR of 15\%-30\% (depending on the FPR of candidates with additional nearby stars), with an indication that FPP increases for larger sizes and shorter periods.

We therefore re-emphasize that lists of $K 2$ candidates and/or validated planets are not currently suitable for the studies of planetary demographics that Kepler so successfully enabled (e.g., Howard et al. 2012; Mulders et al. 2015). The best path forward to enabling such studies would seem to include robust characterization of pipeline detection efficiency, as was done with Kepler (Petigura et al. 2013b; Dressing \& Charbonneau 2015; Christiansen et al. 2015). It may be that such an approach, combined with further refinement of the existing photometry and transit detection pipelines, would allow the first characterization of the frequency of planet occurrence with Galactic environment across the diverse stellar populations probed by $K 2$ 's ecliptic survey.

Barring unexpected technical mishaps, $K 2$ is currently capable of operating through at least $\mathrm{C} 18$. The number of targets observed in these first $K 2$ campaigns contains comparable numbers of targets to later campaigns (with the exception of Campaign 0, which had a duration only roughly half that of the later, $\sim 80$ day campaigns). If $K 2$ continues to observe, based on current discoveries we would expect a planet yield roughly 4-5 times as great as that currently produced. Accounting for the relatively large survey incompleteness revealed by comparing our results to other $K 2$ surveys (Vanderburg et al. 2016), we expect $K 2$ to find anywhere from 500 to 1000 planets over its total mission lifetime. Analysis and follow-up of these systems will occupy exoplanet observers up to the TESS era, and beyond.

For useful suggestions and comments on an early draft we thank Andrew Vanderburg, Knicole Colon, Tim Morton, and Michael Werner. We thank Katherine de Kleer and Imke de Pater for contributing observations, and our many, many telescope operators and observing assistants for helping us obtain such a wealth of data.

This paper includes data collected by the $K 2$ mission. Funding for the $K 2$ mission is provided by the NASA Science Mission directorate.

This work was performed in part under contract with the Jet Propulsion Laboratory (JPL) funded by NASA through the Sagan Fellowship Program executed by the NASA Exoplanet Science Institute. E.A.P. acknowledges support by NASA through a Hubble Fellowship grant awarded by the Space Telescope Science Institute, which is operated by the Association of Universities for Research in Astronomy, Inc., for NASA, under contract NAS 5-26555. The Research of JES was supported by an appointment to the NASA Postdoctoral Program at the NASA Ames Research Center, administered by Universities Space Research Association under contract with NASA. B.J.F. was supported by the National Science Foundation Graduate Research Fellowship under grant No. 2014184874. Any opinion, findings, and conclusions or recommendations expressed in this material are those of the authors and do not necessarily reflect the views of the National Science Foundation. A.J. acknowledges support from FONDECYT project 1130857, BASAL CATA PFB-06, and from the Ministry for the Economy, Development, and Tourism's Programa Iniciativa Científica Milenio through grant IC 120009, awarded to the Millennium Institute of Astrophysics (MAS).

Some of the data presented herein were obtained at the W.M. Keck Observatory (which is operated as a scientific partnership among Caltech, UC, and NASA) and at the Infrared Telescope Facility (IRTF, operated by UH under NASA contract NNH14CK55B). The authors wish to recognize and acknowledge the very significant cultural role and reverence that the summit of Maunakea has always had within the indigenous Hawaiian community. We are most fortunate to have the opportunity to conduct observations from this mountain.

C.B. acknowledges support from the Alfred P. Sloan Foundation. The Robo-AO system was developed by the collaborating partner institutions, the California Institute of Technology and the Inter-University Centre for Astronomy and Astrophysics, and with the support of the National Science Foundation under grant Nos. AST-0906060, AST-0960343, and AST-1207891, the Mt. Cuba Astronomical Foundation, and by a gift from Samuel Oschin.

The Pan-STARRS1 Surveys (PS1) have been made possible through the contributions of the Institute for Astronomy, the University of Hawaii, the Pan-STARRS Project Office, the Max Planck Society and its participating institutes, the Max 
Planck Institute for Astronomy, Heidelberg and the Max Planck Institute for Extraterrestrial Physics, Garching, The Johns Hopkins University, Durham University, the University of Edinburgh, Queen's University Belfast, the HarvardSmithsonian Center for Astrophysics, the Las Cumbres Observatory Global Telescope Network Incorporated, the National Central University of Taiwan, the Space Telescope Science Institute, the National Aeronautics and Space Administration under grant No. NNX08AR22G issued through the Planetary Science Division of the NASA Science Mission Directorate, the National Science Foundation under grant No. AST-1238877, the University of Maryland, and Eotvos Lorand University (ELTE).

Facilities: APF (Levy), Kepler, K2, Keck:I (HIRES), Keck:II (NIRC2), IRTF (SpeX), Palomar:Hale (PALM-3000/ PHARO), Palomar:1.5 m (Robo-AO), Gemini:North (DSSI), Gemini:South (NIRI), LBT (LMIRCam).

\section{REFERENCES}

Adams, E. R., Ciardi, D. R., Dupree, A. K., et al. 2012, AJ, 144, 42 Adams, E. R., Jackson, B., \& Endl, M. 2016, AJ, 152, 47

Aller, K. M., Kraus, A. L., Liu, M. C., et al. 2013, ApJ, 773, 63

Almenara, J. M., Astudillo-Defru, N., Bonfils, X., et al. 2015, A\&A, 581, L7 Angus, R., Foreman-Mackey, D., \& Johnson, J. A. 2016, ApJ, 818, 109 Armstrong, D. J., Kirk, J., Lam, K. W. F., et al. 2016, MNRAS, 456, 2260 Armstrong, D. J., Santerne, A., Veras, D., et al. 2015, A\&A, 582, A33

Baranec, C., Riddle, R., Law, N. M., et al. 2013, Journal of Visualized Experiments, 72, e50021

Baranec, C., Riddle, R., Law, N. M., et al. 2014, ApJL, 790, L8

Baranne, A., Mayor, M., \& Poncet, J. L. 1979, VA, 23, 279

Barros, S. C. C., Almenara, J. M., Demangeon, O., et al. 2015, MNRAS, 454, 4267

Becker, J. C., Vanderburg, A., Adams, F. C., Rappaport, S. A., \& Schwengeler, H. M. 2015, ApJL, 812, L18

Beichman, C., Livingston, J., Werner, M., et al. 2016, ApJ, 822, 39

Borucki, W. J., Koch, D. G., Basri, G., et al. 2011, ApJ, 736, 19

Boyajian, T. S., von Braun, K., van Belle, G., et al. 2012, ApJ, 757, 112

Christiansen, J. L., Clarke, B. D., Burke, C. J., et al. 2015, ApJ, 810, 95

Ciardi, D. R., Beichman, C. A., Horch, E. P., \& Howell, S. B. 2015, ApJ, 805,16

Coelho, P., Barbuy, B., Meléndez, J., Schiavon, R. P., \& Castilho, B. V. 2005, A\&A, 443, 735

Collier Cameron, A., Wilson, D. M., West, R. G., et al. 2007, MNRAS, 380,1230

Coughlin, J. L., Mullally, F., Thompson, S. E., et al. 2016, ApJS, 224, 12

Crossfield, I. J. M., Petigura, E., Schlieder, J. E., et al. 2015, ApJ, 804, 10

Dai, F., Winn, J. N., Albrecht, S., et al. 2016, ApJ, 823, 115

David, T. J., Hillenbrand, L. A., Cody, A. M., Carpenter, J. M., \& Howard, A. W. 2016a, ApJ, 816, 21

David, T. J., Hillenbrand, L. A., Petigura, E. A., et al. 2016b, Natur, 534, 658 Dekany, R., Roberts, J., Burruss, R., et al. 2013, ApJ, 776, 130

Díaz, R. F., Almenara, J. M., Santerne, A., et al. 2014, MNRAS, 441, 983

Dotter, A., Chaboyer, B., Jevremović, D., et al. 2008, ApJS, 178, 89

Dressing, C. D., \& Charbonneau, D. 2015, ApJ, 807, 45

Espinoza, N., et al. 2016, arXiv:1601.07635

Feiden, G. A., \& Chaboyer, B. 2012, ApJ, 757, 42

Foreman-Mackey, D., Hogg, D. W., Lang, D., \& Goodman, J. 2012, arXiv: 1202.3665

Foreman-Mackey, D., Montet, B. T., Hogg, D. W., et al. 2015, ApJ, 806, 215

Fulton, B. J., Howard, A. W., Winn, J. N., et al. 2013, ApJ, 772, 80

Greene, T. P., Line, M. R., Montero, C., et al. 2016, ApJ, 817, 17

Grziwa, S., Gandolfi, D., Csizmadia, S., et al. 2015, arXiv:1510.09149

Hayward, T. L., Brandl, B., Pirger, B., et al. 2001, PASP, 113, 105

Hellier, C., Anderson, D. R., Collier Cameron, A., et al. 2012, MNRAS, 426, 739

Hirano, T., Fukui, A., Mann, A. W., et al. 2016, ApJ, 820, 41

Hodapp, K.-W., Irwin, E. M., Yamada, H., et al. 2003, Proc. SPIE, 4841, 869
Horch, E. P., Howell, S. B., Everett, M. E., \& Ciardi, D. R. 2012, AJ, 144, 165 Horch, E. P., Veillette, D. R., Baena Gallé, R., et al. 2009, AJ, 137, 5057 Howard, A. W., Marcy, G. W., Bryson, S. T., et al. 2012, ApJS, 201, 15 Howard, A. W., Marcy, G. W., Johnson, J. A., et al. 2010, Sci, 330, 653 Howell, S. B., Rowe, J. F., Bryson, S. T., et al. 2012, ApJ, 746, 123 Howell, S. B., Sobeck, C., Haas, M., et al. 2014, PASP, 126, 398 Huang, C. X., Hartman, J. D., Bakos, G. Á., et al. 2015, AJ, 150, 85 Huber, D., Bryson, S. T., Haas, M. R., et al. 2016, ApJS, 224, 2

Huber, D., Ireland, M. J., Bedding, T. R., et al. 2012, ApJ, 760, 32 Johnson, M. C., Gandolfi, D., Fridlund, M., et al. 2016, AJ, 151, 171 Jordán, A., Brahm, R., Bakos, G. Á., et al. 2014, AJ, 148, 29

Kaiser, N., Aussel, H., Burke, B. E., et al. 2002, Proc. SPIE, 4836, 154 Kaufer, A., \& Pasquini, L. 1998, Proc. SPIE, 3355, 844

Kolbl, R., Marcy, G. W., Isaacson, H., \& Howard, A. W. 2015, AJ, 149, 18 Kraus, A. L., \& Hillenbrand, L. A. 2007, AJ, 134, 2340

Kreidberg, L. 2015, PASP, 127, 1161

LaCourse, D. M., Jek, K. J., Jacobs, T. L., et al. 2015, MNRAS, 452, 3561

Law, N. M., Morton, T., Baranec, C., et al. 2014, ApJ, 791, 35

Leisenring, J. M., Skrutskie, M. F., Hinz, P. M., et al. 2012, Proc. SPIE, 8446, $84464 \mathrm{~F}$

Lépine, S., \& Shara, M. M. 2005, AJ, 129, 1483

Lillo-Box, J., Demangeon, O., Santerne, A., et al. 2016, arXiv:1601.07635

Lissauer, J. J., Marcy, G. W., Rowe, J. F., et al. 2012, ApJ, 750, 112

Maldonado, J., Affer, L., Micela, G., et al. 2015, A\&A, 577, A132

Mann, A. W., Gaidos, E., Mace, G. N., et al. 2016, ApJ, 818, 46

Marigo, P., Girardi, L., Bressan, A., et al. 2008, A\&A, 482, 883

Montet, B. T., Morton, T. D., Foreman-Mackey, D., et al. 2015, ApJ, 809, 25

Morton, T. D. 2012, ApJ, 761, 6

Morton, T. D. 2015, VESPA: False Positive Probabilities Calculator, Astrophysics Source Code Library, ascl:1503.011

Morton, T. D., Bryson, S. T., Coughlin, J. L., et al. 2016, ApJ, 822, 86

Mulders, G. D., Pascucci, I., \& Apai, D. 2015, ApJ, 814, 130

Narita, N., Hirano, T., Fukui, A., et al. 2015, ApJ, 815, 47

Neveu-VanMalle, M., Queloz, D., Anderson, D. R., et al. 2016, A\&A, 586, A93

Osborn, H. P., Armstrong, D. J., Brown, D. J. A., et al. 2016, MNRAS, 457, 2273

Parviainen, H., \& Aigrain, S. 2015, MNRAS, 453, 3821

Pecaut, M. J., \& Mamajek, E. E. 2013, ApJS, 208, 9

Petigura, E. A. 2015, arXiv:1510.03902

Petigura, E. A., Howard, A. W., \& Marcy, G. W. 2013a, PNAS, 110, 19273

Petigura, E. A., Howard, A. W., Lopez, E. D., et al. 2016, ApJ, 818, 36

Petigura, E. A., Marcy, G. W., \& Howard, A. W. 2013b, ApJ, 770, 69

Petigura, E. A., Schlieder, J. E., Crossfield, I. J. M., et al. 2015, ApJ, 811, 102

Queloz, D. 1995, in IAU Symp. 167, New Developments in Array Technology and Applications, ed. A. G. D. Philip, K. Janes, \& A. R. Upgren, 221

Ricker, G. R., Winn, J. N., Vanderspek, R., et al. 2014, Proc. SPIE, 9143 20

Rodriguez, D. R., Zuckerman, B., Kastner, J. H., et al. 2013, ApJ, 774, 101 Rowe, J. F., Bryson, S. T., Marcy, G. W., et al. 2014, ApJ, 784, 45 Sanchis-Ojeda, R., Rappaport, S., Pallé, E., et al. 2015, ApJ, 812, 112 Santerne, A., Díaz, R. F., Almenara, J.-M., et al. 2015, MNRAS, 451, 2337 Santerne, A., Hébrard, G., Lillo-Box, J., et al. 2016a, ApJ, 824, 55 Santerne, A., Moutou, C., Tsantaki, M., et al. 2016b, A\&A, 587, A64 Schlieder, J. E., Crossfield, I. J. M., Petigura, E. A., et al. 2016, ApJ, 818, 87 Schmitt, J. R., Tokovinin, A., Wang, J., et al. 2016, AJ, 151, 159 Sinukoff, E., Howard, A. W., Petigura, E., et al. 2016, ApJ, 827, 78 Stassun, K. G., Pepper, J. A., Paegert, M., De Lee, N., \& Sanchis-Ojeda, R. 2014, arXiv: 1410.6379

Ter Braak, C. J. F. 2006, Statistics and Computing, 16, 239

Teske, J. K., Everett, M. E., Hirsch, L., et al. 2015, AJ, 150, 144

Torres, G., Fischer, D. A., Sozzetti, A., et al. 2012, ApJ, 757, 161

Torres, G., Fressin, F., Batalha, N. M., et al. 2011, ApJ, 727, 24

Valenti, J. A., \& Fischer, D. A. 2005, ApJS, 159, 141

Van Cleve, J. E., Howell, S. B., Smith, J. C., et al. 2016, PASP, 128, 075002 Van Eylen, V., Albrecht, S., Gandolfi, D., et al. 2016b, arXiv:1605.09180

Van Eylen, V., Nowak, G., Albrecht, S., et al. 2016a, ApJ, 820, 56 Vanderburg, A., \& Johnson, J. A. 2014, PASP, 126, 948

Vanderburg, A., Latham, D. W., Buchhave, L. A., et al. 2016, ApJS, 222, 14 Vanderburg, A., Montet, B. T., Johnson, J. A., et al. 2015, ApJ, 800, 59

Vogt, S. S., Allen, S. L., Bigelow, B. C., et al. 1994, Proc. SPIE, 2198, 362 Zenteno, A., Forster, F., Rest, A., et al. 2015, ATel, 7664

Ziegler, C., Law, N. M., Morton, T., et al. 2016, arXiv:1605.03584 